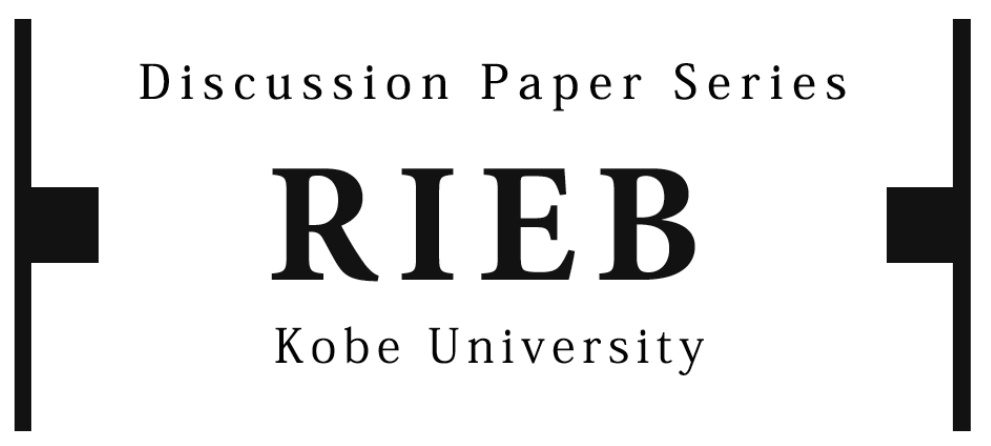

DP2015-27

\title{
Spatial Dependence in Regional Business Cycles: Evidence from Mexican States
}

\author{
Keisuke KONDO
}

Revised June 15, 2021

*This Discussion Paper won the Kanematsu Fellowship Prize (FY 2013).

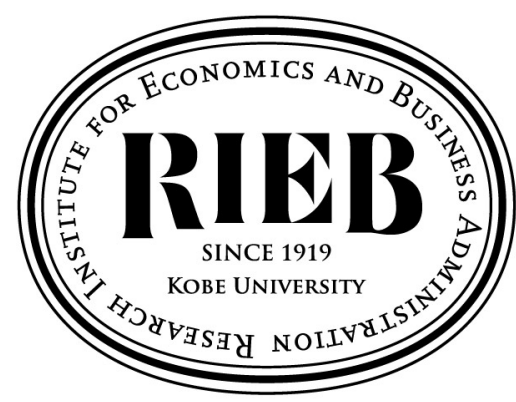

Research Institute for Economics and Business Administration Kobe University 


\title{
Spatial Dependence in Regional Business Cycles: Evidence from Mexican States*
}

\author{
Keisuke Kondo ${ }^{\dagger}$ \\ First Version: January 7, 2013 \\ This Version: June 14, 2021
}

\begin{abstract}
This study investigates how regional business cycles are spatially dependent in Mexico by developing a Markov switching model with a spatial autoregressive process. The Markov switching model with two regimes distinguishes business cycles between expansion and recession phases (i.e., high- and low-growth rate regimes). The objective of this study is twofold. First, this study aims to identify which states transitioned from expansion to recession during the Great Recession in 2008-2009. Second, it numerically examines the extent to which states that experienced this transition caused a deterioration in neighboring states' economies. Employing Bayesian inference for the Markov switching model with quarterly data of state economic activity during the period 2003:Q1-2015:Q4, this study finds that Mexican states with higher manufacturing sector shares tended to be in recession during the Great Recession. Although some states experienced economic downturns in this period, they were not in a recessionary regime. This study also finds that business cycles across states were spatially dependent during the Great Recession. The numerical simulations of spatial spillover effects suggest that states that regimeswitched from expansion to recession during the Great Recession caused a reduction in the quarterly growth rate of their nearest neighboring economies by an average of 0.26 percentage points.
\end{abstract}

Keywords: Spatial Dependence, Spatial Spillover Effects, Regional Business Cycles, Markov Switching Model, Markov Chain Monte Carlo

JEL classifications: C33, E32

\footnotetext{
* I would like to specially thank Alfredo Erquizio Espinal and Kensuke Teshima for their insightful comments and helpful suggestions. I also thank Arthur Getis, Nobuaki Hamaguchi, Yoichi Matsubayashi, Akio Namba, Tatsuyoshi Okimoto, Sergio J. Rey, Andrzej Torój, Carlos Urrutia, and participants at the 2013 Spring Meeting of the Japanese Economic Association, the Rokko Forum at Kobe University, and the 53rd annual meeting of the Western Regional Science Association for their useful comments and suggestions. I acknowledge use of computer routines described in Kim and Nelson (1999b). Naturally, any remaining errors are my own. Furthermore, I am grateful for the benefits received during my stay at the Instituto Tecnológico Autónomo de México in 2012-2013. This research was carried out under a scholarship granted by the Government of Mexico, through the Ministry of Foreign Affairs of Mexico. This paper received the 2013 Kanematsu Fellowship Award from the Research Institute for Economics and Business Administration, Kobe University. Supplementary information is available on GitHub (URL: https://keisukekondokk.github.io/).

$\dagger$ Research Institute of Economy, Trade and Industry (RIETI). 1-3-1 Kasumigaseki, Chiyoda-ku, Tokyo, 100-8901, Japan.

(e-mail: kondo-keisuke@rieti.go.jp)
} 


\section{Introduction}

As conditions in regional economies do not necessarily coincide with national economic conditions, regional business cycles tend to be highly heterogeneous. However, spatial proximity seems to characterize similarity among regional business cycles. Thus, in light of the interdependence of regional economies, we focus on spatial spillover (or neighborhood) effects across regional business cycles. In such an economic situation, a region-specific shock may cause deterioration in the economic conditions of neighboring economies. In recent years, the importance of conducting spatial analyses of economic activities has been emphasized from economic stability and growth perspectives (e.g., World Bank, 2009). We therefore investigate spatial dependence within regional business cycles.

To analyze spatial dependence in regional business cycles and spatial spillover effects, we integrate a spatially lagged dependent variable into a Markov switching model. The Markov switching model with two regimes endogenously distinguishes business cycles between expansion and recession phases (i.e., high- and low-growth rate regimes). Our integrated approach from the Markov switching model and spatial econometrics thus enables us to identify which states experienced a transition from expansion to recession as well as to numerically examine the extent to which states that experienced such a transition caused a deterioration in their neighboring states' economies.

Regional business cycles are not perfectly uniform, and thus discussions of the national business cycle are not directly applicable. For example, in applying the Markov switching model proposed by Hamilton (1989), Owyang et al. (2005) found that business cycles across US states differed considerably in terms of expansionary and recessionary phases. Furthermore, Owyang et al. (2008) investigated business cycles at the US city level and drew similar conclusions. To explain the similarities and differences among regional business cycles, Hamilton and Owyang (2012) developed a Markov switching model based on the rationale that administrative units do not necessarily coincide with economic zones. In their model, US states are endogenously grouped into clusters that share similar economic characteristics by identifying common factors across states. Therefore, the authors' focus is on regional recessions within each group. ${ }^{1}$ They found that states that have a relatively high share of oil production or agriculture were more likely to be in recession than other US states. By contrast, we focus on the spatial association of regional business cycles because the empirical results of Owyang et al. (2005) imply that state recessions appear to occur among states located close to each other. Therefore, this study emphasizes spatial dependence in regional business cycles.

This study uses quarterly data of state economic activity for Mexico. Previous studies, such as those by Owyang et al. (2008) and Hamilton and Owyang (2012), used employment data for business cycle analysis because monthly or quarterly economic activity data were not available at the city or state level in the US. However, employment data may not accurately reflect contemporaneous economic conditions because of labor market rigidities. By contrast, in Mexico, the economic activity data of each state have been collected quarterly and published as an official indicator since the early 2000s. ${ }^{2}$ Capitalizing on these data, we investigate how a state recession, caused by the global economic crisis

\footnotetext{
${ }^{1}$ The approach of Hamilton and Owyang (2012) can identify regional common factors of business cycles within a Markov switching model. Another major approach to regional business cycles is to estimate a dynamic factor model. For example, see Kose et al. (2003), Owyang et al. (2009), and Hirata et al. (2013).

${ }^{2}$ Note that business cycles should involve co-movement of a wide range of economic activities such as output, employment, and sales (Stock and Watson, 1989). Following Burns and Mitchell (1946), as emphasized by Stock and Watson (1989), it is imprecise to define
} 
of 2008-2009, spread to the neighboring states through the domestic economic network.

Recent studies have attempted to describe business cycles across Mexican states. Synchronization between the Mexican and US economies has long attracted economists' attention. For example, Chiquiar and Ramos-Francia (2005) investigated business cycle synchronization between the Mexican and US manufacturing sectors. Although this type of analysis does not account for regional economic activities within Mexico, it offers suggestive evidence that Mexican states with stronger connections with the US exhibit business cycle synchronization within Mexico. In this sense, Mejía-Reyes and Campos-Chávez (2011) investigated synchronization between Mexican states and US manufacturing production, finding that Mexican states with a relatively high share of manufacturing are more strongly affected by US manufacturing production. Mejía-Reyes et al. (2018) also investigated synchronization between Mexican states and the US and found that FDI also had significant and positive effects on the synchronization between Mexican states and the US.

To determine the phases of regional business cycles and investigate regional differences during the economic crisis of 2008-2009, Erquizio-Espinal (2010) calculated the coincidence index across Mexican states, in the spirit of Burns and Mitchell (1946). He found that the border states, which are closely related to the US economy, were more strongly affected by the US recession. As emphasized by Mejía-Reyes and Erquizio-Espinal (2012), one of the unique causes of the Great Recession of 2008-2009 in Mexico was the transmission of the business cycle shock from the US. This is consistent with Torres and Vela (2003) and Sosa (2008), who investigated business cycle synchronization between the US and Mexico before the Great Recession of 2008-2009 in Mexico. However, it remains unclear how a recession in the US economy spread across Mexican states. Although some states did not experience sharp declines in quarterly state economic activity during the Great Recession, recessions in states with stronger links to the US economy would have caused a slowdown in their neighboring states' economies. By considering domestic factors in regional business cycles, this study sheds new light on this transmission mechanism. ${ }^{3}$

This study contributes to the literature by demonstrating how a region-specific shock propagates to neighboring economies. In the literature on spatial econometrics, Anselin (1988) estimated spatial econometric models that were able to account for spatial dependence and heterogeneity. In this study, we connect regional interdependence with business cycles in a macroeconomic time-series analysis by estimating the Markov switching model proposed by Hamilton (1989). ${ }^{4}$ We estimating the model using the Bayesian Markov chain Monte Carlo (MCMC) method. ${ }^{5}$

business cycles only in terms of fluctuations in either GDP or employment. Nevertheless, a Markov switching model that uses GDP growth rates provides insights into business cycles by estimating unobservable expansionary and recessionary regimes.

${ }^{3}$ Delajara (2011) investigated co-movement across Mexican states during the recession period of 2008-2009. He suggested the possibility of geographical propagation, although he did not provide direct evidence. This study provides the evidence to support his discussion.

${ }^{4}$ To the best of my knowledge, Ohtsuka (2010) is the first to introduce a spatial autoregressive process into a standard Markov switching model, discovering that business cycles across Japanese regions are spatially dependent.

${ }^{5}$ An MCMC estimation methodology for a Markov switching model was first suggested by Albert and Chib (1993). However, there were questions related to how discrete, hidden variables ought to be sampled. By developing the single-move Gibbs sampling initially proposed by Albert and Chib (1993), Kim and Nelson (1998, 1999a, b) improved sampling efficiency by using multi-move Gibbs sampling for hidden variables. In the literature on regional business cycles, Owyang et al. (2005) and Owyang et al. (2008) also adopted the method proposed by Kim and Nelson (1998, 1999a, 1999b) for model estimation. 
LeSage and Pace (2009) described a Bayesian estimation methodology for spatial econometric models and applied the Metropolis-Hasting (MH) algorithm to estimate a parameter measuring spatial dependence. Hence, their study is notable in that the estimation method used is characterized by the MH algorithm, rather than by Gibbs sampling. ${ }^{6}$ In this study, we develop the Bayesian MCMC estimation method proposed by Kim and Nelson (1998, 1999a, b) by introducing a spatial autoregressive process.

Employing Bayesian inference for the Markov switching model with quarterly data of state economic activity during the period 2003:Q1-2015:Q4, this study finds that Mexican states with a higher manufacturing share tended to be in recession during the Great Recession. This study also finds that business cycles across states were spatially dependent during the Great Recession. The numerical simulations of spatial spillover effects suggest that states that experienced a transition from expansion to recession during the Great Recession caused a decrease in the quarterly growth rate of the nearest neighboring economy by an average of 0.26 percentage points.

The remainder of this paper is organized as follows. In Section 2, we describe the Markov switching model, including a discussion of the spatially lagged dependent variable. In Section 3, we present the Bayesian estimation procedure using MCMC. In Section 4, we present the data. In Section 5, we discuss the estimation results. In Section 6 , we provide a numerical simulation of the spatial spillover effects of a regional recession. Finally, In Section 7, we present our conclusions.

\section{Markov Switching Model with Spatial Lag}

Let $y_{t, n}$ denote the growth rate of an indicator of economic activity for region $n$ at date $t$. Using vector notation, we denote $\boldsymbol{y}_{t}=\left(y_{t, 1}, y_{t, 2}, \ldots, y_{t, N}\right)^{\top}$ as an $N \times 1$ vector, where $N$ represents the number of regions. Let $\boldsymbol{s}_{t}=$ $\left(s_{t, 1}, s_{t, 2}, \ldots, s_{t, N}\right)^{\top}$ denote an $N \times 1$ vector of the indicator variable that follows a Markov chain. If $s_{t, n}=1$, then region $n$ is in the expansion phase at date $t$, whereas $s_{t, n}=0$ means that region $n$ is in the recession phase at date $t$. Based on Hamilton (2008), a multi-regional Markov switching model involving a first-order autoregressive process, $\mathrm{AR}(1)$, of the dependent variable can be described as follows:

$$
\boldsymbol{y}_{t}=\boldsymbol{\Phi} \boldsymbol{y}_{t-1}+\boldsymbol{\mu}_{0} \odot\left(\boldsymbol{\iota}_{N}-\boldsymbol{s}_{t}\right)+\boldsymbol{\mu}_{1} \odot \boldsymbol{s}_{t}+\boldsymbol{\varepsilon}_{t}, \quad t=2, \ldots, T
$$

where $\boldsymbol{\Phi}=\operatorname{diag}\left(\phi_{1}, \ldots, \phi_{N}\right)$ is an $N \times N$ diagonal matrix with parameter $\phi_{i}$ that measures the temporal dependence in the dependent variable for state $n ; \boldsymbol{\mu}_{0}=\left(\mu_{1,0}, \mu_{2,0}, \ldots, \mu_{N, 0}\right)^{\top}$ is an $N \times 1$ vector of the average growth rate in the recession phase; $\boldsymbol{\mu}_{1}=\left(\mu_{1,1}, \mu_{2,1}, \ldots, \mu_{N, 1}\right)^{\top}$ is an $N \times 1$ vector of the average growth rate in the expansion phase; $\boldsymbol{\iota}_{N}$ is an $N \times 1$ vector whose all elements are ones; $\varepsilon_{t}=\left(\varepsilon_{t, 1}, \varepsilon_{t, 2}, \ldots, \varepsilon_{t, N}\right)^{\top}$ is an $N \times 1$ vector of error terms that follow i. i. d. $\mathrm{N}(\mathbf{0}, \boldsymbol{\Omega})$; and $\odot$ denotes element-by-element multiplication. Model (1) is a baseline model that does not include spatial dependence in regional business cycles and can be estimated separately in each region.

In this study, we introduce a spatially lagged dependent variable into a Markov switching model with AR(1). Let

${ }^{6}$ This difference implies the need for modifications in the discussion on model selection. See Chib (1995) and Chib and Jeliazkov (2001) for more detailed discussions. 
$\boldsymbol{W}$ denote an $N \times N$ spatial weight matrix (SWM). Thus, $\boldsymbol{W} \boldsymbol{y}_{t}$ indicates an $N \times 1$ vector of the spatially lagged dependent variable. The Markov switching model involving the spatial lag is denoted as follows:

$$
\boldsymbol{y}_{t}=\rho \boldsymbol{W} \boldsymbol{y}_{t}+\boldsymbol{\Phi} \boldsymbol{y}_{t-1}+\boldsymbol{\mu}_{0} \odot\left(\boldsymbol{\iota}_{N}-\boldsymbol{s}_{t}\right)+\boldsymbol{\mu}_{1} \odot \boldsymbol{s}_{t}+\boldsymbol{\varepsilon}_{t}
$$

where $\rho$ is a parameter measuring spatial dependence in the dependent variable.

In model (2), we impose the restriction that $\mu_{n, 1}>\mu_{n, 0}, n=1,2, \ldots, N$ because the average growth rate in the expansion phase is higher than that in the recession phase. We assume that error terms are independent across regions, that is, $\boldsymbol{\Omega}=\operatorname{diag}\left(\sigma_{1}^{2}, \sigma_{2}^{2}, \ldots, \sigma_{N}^{2}\right)$ is an $N \times N$ diagonal matrix. We also assume that $s_{t, n}$ follows a first-order twostate Markov chain, indicating that the transition probabilities are $\operatorname{Pr}\left(s_{t, n}=i \mid s_{t-1, n}=j\right)=p_{n, j i}, i, j=0,1$.

The SWM is a row-standardized matrix constructed from geographical information based on contiguity or distance between regions. The SWM describes a spatial spillover network across regions. The spatial autoregressive parameter $\rho$ lies in the interval of the inverses of the minimum and maximum eigenvalues of the SWM. As shown in Ord (1975), the row-standardized SWM yields +1 as the largest eigenvalue. Therefore, we impose the restriction $1 / \omega_{\min }<\rho<$ 1 , where $\omega_{\min }$ is the smallest eigenvalue of the SWM. ${ }^{7}$

Our Markov switching model includes a spatially lagged dependent variable $\boldsymbol{W} \boldsymbol{y}_{t}$. Therefore, the coefficient parameter $\rho$ measures spatial dependence in regional business cycles. An advantage of this model is that it allows us to numerically simulate the spatial spillover effects for a particular region-specific shock. As described in Anselin (2003), if $\rho \neq 0$, spatial spillover effects exist. Thus, based on model (2), we numerically investigate how Mexican states that experienced a transition from expansion to recession during the period 2008-2009 affected their neighboring states' economies.

We implicitly assume that observed spatial dependence in regional business cycles results from the spatial network structures in economic activities. Therefore, the numerical simulations of the spatial spillover effects aim to recover the potential spatial impacts that occurred in the real economy. However, spatial dependence in regional business cycles can be observed even when the external common shocks occur independently among regions. Thus, the statistical significance of the spatial autoregressive parameter $\rho$ does not necessarily ensure the spatial network structure in economic activities. The spatial network structure generating spatial spillover effects across the Mexican states is additionally examined in Appendix D.

\section{Bayesian Inference}

Bayesian inference, such as point and interval estimates, is based on a posterior distribution. For convenience of explanation, we define the parameter vector $\boldsymbol{\theta}=\left\{\boldsymbol{\Omega}, \boldsymbol{\mu}, \boldsymbol{\Phi}, \boldsymbol{p}_{11}, \boldsymbol{p}_{00}, \rho\right\}$, where $\boldsymbol{\mu}=\left(\boldsymbol{\mu}_{0}, \boldsymbol{\mu}_{1}\right)^{\top}, \boldsymbol{p}_{11}=$ $\left(p_{1,11}, p_{2,11}, \ldots, p_{N, 11}\right)^{\top}$, and $\boldsymbol{p}_{00}=\left(p_{1,00}, p_{2,00}, \ldots, p_{N, 00}\right)^{\top}$. Let $\boldsymbol{Y}=\left\{y_{t, n}\right\}$ and $\boldsymbol{S}=\left\{s_{t, n}\right\}$ each denote a $T \times N$ matrix. Thus, by using Bayes' theorem, we can derive the following relationship regarding the posterior distribution:

\footnotetext{
${ }^{7}$ For the SWM used in this study, $\omega_{\min }$ always takes a negative value, as it does in most cases. See also Anselin and Bera (1998) for a more detailed discussion.
} 


$$
\pi(\boldsymbol{\theta} \mid \boldsymbol{Y}, \boldsymbol{S}) \propto L(\boldsymbol{Y}, \boldsymbol{S} \mid \boldsymbol{\theta}) \pi(\boldsymbol{\theta}),
$$

where $\pi(\boldsymbol{\theta} \mid \boldsymbol{Y}, \boldsymbol{S})$ is the posterior distribution, $L(\boldsymbol{Y}, \boldsymbol{S} \mid \boldsymbol{\theta})$ is the likelihood function, $\pi(\boldsymbol{\theta})$ is the prior distribution, and $\propto$ represents "is proportional to." An important result here is that the posterior distribution is proportional to the likelihood function multiplied by the prior distribution. We therefore need to specify prior distributions and to derive the likelihood function to conduct the Bayesian inference.

\subsection{Prior Distributions and Likelihood Function}

First, we specify the prior distributions of the parameters. Thus, we use an inverse gamma distribution $\operatorname{IG}(\underline{v} / 2, \underline{\delta} / 2)$ as a prior for $\sigma_{n}^{2}$ as follows:

$$
\pi\left(\sigma_{n}^{2}\right) \propto\left(\frac{1}{\sigma_{n}^{2}}\right)^{\underline{v} / 2+1} \exp \left(\frac{\delta}{2 \sigma_{n}^{2}}\right)
$$

As for a prior for $\boldsymbol{\mu}_{n}=\left(\mu_{n, 0}, \mu_{n, 1}\right)^{\top}$, we adopt a bivariate normal distribution $\mathrm{N}_{2}\left(\underline{\boldsymbol{m}}_{\mu}, \underline{\boldsymbol{M}}_{\mu}\right)$ as follows:

$$
\pi\left(\boldsymbol{\mu}_{n}\right) \propto \exp \left(-\frac{1}{2}\left(\boldsymbol{\mu}_{n}-\underline{\boldsymbol{m}}_{\mu}\right)^{\top} \underline{\boldsymbol{M}}_{\mu}^{-1}\left(\boldsymbol{\mu}_{n}-\underline{\boldsymbol{m}}_{\mu}\right)\right)
$$

where we impose a restriction $\mu_{n, 1}>\mu_{n, 0}$. As for a prior for $\phi_{n}$, we adopt a univariate normal distribution $\mathrm{N}_{1}\left(\underline{m}_{\phi}, \underline{M}_{\phi}\right)$ as follows:

$$
\pi\left(\boldsymbol{\phi}_{n}\right) \propto \exp \left(-\frac{1}{2} \frac{\left(\phi_{n}-\underline{m}_{\phi}\right)^{2}}{\underline{M}_{\phi}}\right) .
$$

Prior distributions for the transition probabilities $p_{n, 11}$ and $p_{n, 00}$ are set to have beta distributions $\operatorname{Beta}\left(\underline{\alpha}_{11}, \underline{\alpha}_{10}\right)$ and $\operatorname{Beta}\left(\underline{\alpha}_{00}, \underline{\alpha}_{01}\right)$, respectively:

$$
\pi\left(p_{n, 11}\right) \propto p_{n, 11}^{\underline{\alpha}_{11}-1}\left(1-p_{n, 11}\right)^{\underline{\alpha}_{10}-1} \quad \text { and } \quad \pi\left(p_{n, 00}\right) \propto p_{n, 00}^{\underline{\alpha}_{00}-1}\left(1-p_{n, 00}\right)^{\underline{\alpha}_{01}-1} .
$$

Finally, we use a uniform distribution $\mathrm{U}\left(1 / \omega_{\min }, 1\right)$ as a prior for $\rho$.

Next, we derive the likelihood function. To evaluate it, we consider two decomposed terms by utilizing $L(\boldsymbol{Y}, \boldsymbol{S} \mid \boldsymbol{\theta})=L(\boldsymbol{Y} \mid \boldsymbol{\theta}, \boldsymbol{S}) p(\boldsymbol{S} \mid \boldsymbol{\theta})$, where $p(\cdot)$ is a probability mass function. From the assumption that $\boldsymbol{\varepsilon}_{t} \sim$ i. i. d. $\mathrm{N}(\mathbf{0}, \boldsymbol{\Omega})$ and by using variable transformation, the likelihood function conditional on $\boldsymbol{S}$ is given by the following:

$$
L(\boldsymbol{Y} \mid \boldsymbol{\theta}, \boldsymbol{S})=\prod_{t=1}^{T}\left[(2 \pi)^{-N / 2}|\boldsymbol{\Omega}|^{-1 / 2}\left|\boldsymbol{I}_{N}-\rho \boldsymbol{W}\right| \exp \left(-\frac{1}{2} \boldsymbol{\varepsilon}_{t}^{\top} \boldsymbol{\Omega}^{-1} \boldsymbol{\varepsilon}_{t}\right)\right],
$$

where $\boldsymbol{I}_{N}$ is an $N \times N$ identity matrix and $\boldsymbol{\varepsilon}_{t}=\left(\boldsymbol{I}_{N}-\rho \boldsymbol{W}\right) \boldsymbol{y}_{t}-\boldsymbol{\Phi} \boldsymbol{y}_{t-1}-\boldsymbol{\mu}_{0} \odot\left(\boldsymbol{\iota}_{N}-\boldsymbol{s}_{t}\right)-\boldsymbol{\mu}_{1} \odot \boldsymbol{s}_{t}$. The 
difficulty is that the hidden variables $\boldsymbol{S}$ are directly unobservable. To evaluate the likelihood function, we follow the methodology of Kim and Nelson (1998, 1999a, b). See Appendix B for more technical details.

\subsection{Posterior Distributions}

Having specified the likelihood function and prior distributions, we are able to conduct the Bayesian inference. From (3), the joint posterior distribution is given by the following:

$$
\pi(\boldsymbol{\theta} \mid \boldsymbol{Y}, \boldsymbol{S}) \propto L(\boldsymbol{Y} \mid \boldsymbol{\theta}, \boldsymbol{S}) p(\boldsymbol{S} \mid \boldsymbol{\theta}) \pi(\rho) \pi(\boldsymbol{\Omega}) \pi(\boldsymbol{\mu}) \pi(\boldsymbol{\Phi}) \pi\left(\boldsymbol{p}_{11}\right) \pi\left(\boldsymbol{p}_{00}\right)
$$

where an independent joint prior distribution across parameters and regions is assumed. For ease of explanation, let us define the following vectors with respect to region $n: \boldsymbol{\mu}_{n}=\left(\mu_{n, 0}, \mu_{n, 1}\right)^{\top}, \boldsymbol{s}_{n}=\left(s_{2, n}, s_{3, n}, \ldots, s_{T, n}\right)^{\top}, \boldsymbol{y}_{n}=$ $\left(y_{2, n}, y_{3, n}, \ldots, y_{T, n}\right)^{\top} \quad, \quad \overline{\boldsymbol{y}}_{n}=\left(\sum_{m=1}^{N} w_{n m} y_{2, m}, \sum_{m=1}^{N} w_{n m} y_{3, m}, \ldots, \sum_{m=1}^{N} w_{n m} y_{T, m}\right)^{\top} \quad, \quad$ and $\quad \tilde{\boldsymbol{y}}_{n}=$ $\left(y_{1, n}, y_{2, n}, \ldots, y_{T-1, n}\right)^{\top}$. However, a difficulty concerning sampling from the posterior distribution arises because a Markov switching model includes hidden variables $\boldsymbol{S}$. Therefore, we present a more detailed discussion for the sampling methodology.

The sampling methodology is based on the conditional posterior distributions. Although we assumed an independent joint prior, the conditional posterior distributions are from the same family of distributions as the priors, except for parameter $\rho .{ }^{8}$ As the conditional posterior distribution for $\rho$ takes an unknown distributional form, we rely on the MH algorithm. However, those for $\boldsymbol{\Omega}, \boldsymbol{\mu}, \boldsymbol{\Phi}, \boldsymbol{p}_{11}$, and $\boldsymbol{p}_{00}$ take known distributional forms, and we thus apply the Gibbs sampler for posterior sampling of these parameters.

We derive conditional posterior distributions distribution for each parameter below. The conditional posterior distribution for $\rho \mid \boldsymbol{Y}, \boldsymbol{S}, \boldsymbol{\mu}, \boldsymbol{\Phi}, \boldsymbol{\Omega}$ is given by the following:

$$
\pi(\rho \mid \boldsymbol{Y}, \boldsymbol{S}, \boldsymbol{\mu}, \boldsymbol{\Phi}, \boldsymbol{\Omega}) \propto \prod_{t=1}^{T}\left[\left|\boldsymbol{I}_{N}-\rho \boldsymbol{W}\right| \exp \left(-\frac{1}{2} \boldsymbol{\varepsilon}_{t}^{\top}(\rho) \boldsymbol{\Omega}^{-1} \boldsymbol{\varepsilon}_{t}(\rho)\right)\right]
$$

where $\boldsymbol{\varepsilon}_{t}(\rho)=\left(\boldsymbol{I}_{N}-\rho \boldsymbol{W}\right) \boldsymbol{y}_{t}-\boldsymbol{\Phi} \boldsymbol{y}_{t-1}-\boldsymbol{\mu}_{0} \odot\left(\boldsymbol{\iota}_{N}-\boldsymbol{s}_{t}\right)-\boldsymbol{\mu}_{1} \odot \boldsymbol{s}_{t}$. Note that $\rho$ is independent of $p_{n, 11}$ and $p_{n, 00}$. As mentioned previously, this is an unknown distributional form. We therefore rely on the MH algorithm. In the literature on spatial econometrics, LeSage and Pace (2009) described a sampling method for a parameter on spatial dependence using the MH algorithm. Our sampling follows their method. See Appendix A for more technical details.

As for the sampling for the other conditional posterior distribution, although there are slight changes, the sampling strategy is the same as the one proposed in Kim and Nelson (1998, 1999a, b). Samples from these conditional posteriors are drawn via the Gibbs sampler. First, the conditional posterior distribution for $\sigma_{n}^{2} \mid \boldsymbol{Y}, \boldsymbol{S}, \boldsymbol{\mu}_{n}, \rho$ takes the following form:

\footnotetext{
${ }^{8}$ The priors for $\boldsymbol{\Omega}, \boldsymbol{\mu}, \boldsymbol{\Phi}, \boldsymbol{p}_{11}, \boldsymbol{p}_{00}$ are conditionally conjugate.
} 


$$
\pi\left(\sigma_{n}^{2} \mid \boldsymbol{Y}, \boldsymbol{S}, \boldsymbol{\mu}_{n}, \phi_{n}, \rho\right) \propto\left(\frac{1}{\sigma_{n}^{2}}\right)^{\bar{v} / 2+1} \exp \left(\frac{\bar{\delta}_{n}}{2 \sigma_{n}^{2}}\right)
$$

where $\bar{v}=\underline{v}+T$ and $\bar{\delta}_{n}=\underline{\delta}+\boldsymbol{\varepsilon}_{n}^{\top} \boldsymbol{\varepsilon}_{n}$. Note that $\sigma_{n}^{2}$ is independent of $p_{n, 11}$ and $p_{n, 00}$. We can see that this conditional posterior for $\sigma_{n}^{2} \mid \boldsymbol{Y}, \boldsymbol{S}, \boldsymbol{\mu}_{n}, \phi_{n}, \rho$ is distributed as an inverse gamma distribution $\operatorname{IG}\left(\bar{v} / 2, \bar{\delta}_{n} / 2\right)$.

Next, the conditional posterior distribution for $\boldsymbol{\mu}_{n} \mid \boldsymbol{Y}, \boldsymbol{S}, \sigma_{n}^{2}, \phi_{n}, \rho$ is obtained as follows:

$$
\pi\left(\boldsymbol{\mu}_{n} \mid \boldsymbol{Y}, \boldsymbol{S}, \sigma_{n}^{2}, \phi_{n}, \rho\right) \propto \exp \left(-\frac{1}{2}\left(\boldsymbol{\mu}_{n}-\overline{\boldsymbol{m}}_{\mu, n}\right)^{\top} \overline{\boldsymbol{M}}_{\mu, n}^{-1}\left(\boldsymbol{\mu}_{\mu, n}-\overline{\boldsymbol{m}}_{\mu, n}\right)\right),
$$

where

$$
\begin{aligned}
\overline{\boldsymbol{M}}_{\mu, n} & =\left(\underline{\boldsymbol{M}}_{\mu}^{-1}+\sigma_{n}^{-2} \boldsymbol{X}_{n}^{\top} \boldsymbol{X}_{n}\right)^{-1}, \\
\overline{\boldsymbol{m}}_{\mu, n} & =\overline{\boldsymbol{M}}_{\mu, n}\left(\underline{\boldsymbol{M}}_{\mu}^{-1} \underline{\boldsymbol{m}}_{\mu}+\sigma_{n}^{-2} \boldsymbol{X}_{n}^{\top}\left(\boldsymbol{y}_{n}-\rho \overline{\boldsymbol{y}}_{n}-\phi_{n} \widetilde{\boldsymbol{y}}_{n}\right)\right), \\
\boldsymbol{X}_{n} & =\left(\boldsymbol{\iota}_{T}-\boldsymbol{s}_{n} \boldsymbol{s}_{n}\right) .
\end{aligned}
$$

Note that $\boldsymbol{\mu}_{n}$ is independent of $p_{n, 11}$ and $p_{n, 00}$. The conditional posterior for $\boldsymbol{\mu}_{n} \mid \boldsymbol{Y}, \boldsymbol{S}, \sigma_{n}^{2}, \phi_{n}, \rho$ is distributed as a bivariate normal distribution with mean $\overline{\boldsymbol{m}}_{\mu, n}$ and variance $\overline{\boldsymbol{M}}_{\mu, n}$, that is, $\mathrm{N}_{2}\left(\overline{\boldsymbol{m}}_{\mu, n}, \overline{\boldsymbol{M}}_{\mu, n}\right)$.

The conditional posterior distribution for $\phi_{n} \mid \boldsymbol{Y}, \boldsymbol{S}, \sigma_{n}^{2}, \boldsymbol{\mu}_{n}, \rho$ is obtained as follows:

$$
\pi\left(\phi_{n} \mid \boldsymbol{Y}, \boldsymbol{S}, \sigma_{n}^{2}, \boldsymbol{\mu}_{n}, \rho\right) \propto \exp \left(-\frac{1}{2} \frac{\left(\phi_{n}-\bar{m}_{\phi, n}\right)^{2}}{\bar{M}_{\phi, n}}\right)
$$

where

$$
\begin{aligned}
& \bar{M}_{\phi, n}=\left(\underline{M}_{\phi}^{-1}+\sigma_{n}^{-2} \widetilde{\boldsymbol{y}}_{n}^{\top} \widetilde{\boldsymbol{y}}_{n}\right)^{-1}, \\
& \bar{m}_{\phi, n}=\bar{M}_{\phi, n}\left(\underline{M}_{\phi}^{-1} \underline{m}_{\phi}+\sigma_{n}^{-2} \widetilde{\boldsymbol{y}}_{n}^{\top}\left(\boldsymbol{y}_{n}-\rho \overline{\boldsymbol{y}}_{n}-\mu_{0, n} s_{t n}-\mu_{1, n}\left(1-s_{t n}\right)\right)\right) .
\end{aligned}
$$

Note that $\phi_{n}$ is independent of $p_{n, 11}$ and $p_{n, 00}$. The conditional posterior for $\boldsymbol{\phi}_{n} \mid \boldsymbol{Y}, \boldsymbol{S}, \sigma_{n}^{2}, \boldsymbol{\mu}_{n}, \rho$ is distributed as a univariate normal distribution with mean $\bar{m}_{\phi, n}$ and variance $\bar{M}_{\phi, n}$, that is, $\mathrm{N}_{1}\left(\bar{m}_{\phi, n}, \bar{M}_{\phi, n}\right)$.

Finally, we derive the conditional posterior distributions for $p_{n, 11} \mid \boldsymbol{Y}, \boldsymbol{S}$ and $p_{n, 00} \mid \boldsymbol{Y}, \boldsymbol{S}$ that are given by the following:

$$
\pi\left(p_{n, 11} \mid \boldsymbol{Y}, \boldsymbol{S}\right) \propto p_{n, 11}^{\bar{\alpha}_{11}-1}\left(1-p_{n, 11}\right)^{\bar{\alpha}_{10}-1} \quad \text { and } \quad \pi\left(p_{n, 00} \mid \boldsymbol{Y}, \boldsymbol{S}\right) \propto p_{n, 00}^{\bar{\alpha}_{00}-1}\left(1-p_{n, 00}\right)^{\bar{\alpha}_{01}-1}
$$

where $\bar{\alpha}_{11}=\underline{\alpha}_{11}+n_{11}, \quad \bar{\alpha}_{10}=\underline{\alpha}_{10}+n_{10}, \bar{\alpha}_{00}=\underline{\alpha}_{00}+n_{00}, \bar{\alpha}_{01}=\underline{\alpha}_{01}+n_{01}$, and $n_{j i}$ is the number of transitions from state $j$ to state $i$. Note that $p_{n, 11}$ and $p_{n, 00}$ are independent of $\sigma_{n}^{2}, \boldsymbol{\mu}_{n}$, and $\rho$. We see that $p_{n, 11} \mid \boldsymbol{Y}, \boldsymbol{S}$ and $p_{n, 00} \mid o l Y, \boldsymbol{S}$ follow the beta distributions $\operatorname{Beta}\left(\bar{\alpha}_{11}, \bar{\alpha}_{10}\right)$ and $\operatorname{Beta}\left(\bar{\alpha}_{00}, \bar{\alpha}_{01}\right)$, respectively. 


\subsection{Drawing Parameters from Posterior Distributions}

We conduct the Bayesian inference via multiple-block MH sampling. ${ }^{9}$ Besides parameters, a Markov switching model includes hidden variables $\boldsymbol{S}$. Following Kim and Nelson (1998, 1999a, b), we use multi-move Gibbs sampling for drawing $\boldsymbol{s}_{n}$. In summary, our sampling algorithm is as follows:

1. Set hyperparameters and the initial parameter values.

2. Draw $\boldsymbol{s}_{n}^{(g)} \mid \boldsymbol{Y}, \boldsymbol{\theta}^{(g-1)}(n=1, \ldots, N)$ from the multi-move Gibbs sampling. ${ }^{10}$

3. Draw $p_{n, 11}^{(g)} \mid \boldsymbol{Y}, \boldsymbol{s}_{n}^{(g)}(n=1, \ldots, N)$ from $\operatorname{Beta}\left(\bar{\alpha}_{11}, \bar{\alpha}_{10}\right)$.

4. Draw $p_{n, 00}^{(g)} \mid \boldsymbol{Y}, \boldsymbol{s}_{n}^{(g)}(n=1, \ldots, N)$ from $\operatorname{Beta}\left(\bar{\alpha}_{00}, \bar{\alpha}_{01}\right)$.

5. $\quad \operatorname{Draw} \sigma_{n}^{2,(g)} \mid \boldsymbol{Y}, \boldsymbol{s}_{n}^{(g)}, \boldsymbol{\mu}_{n}^{(g-1)}, \phi_{n}^{(g-1)}, \rho^{(g-1)}(n=1, \ldots, N)$ from $\operatorname{IG}(\bar{v} / 2, \bar{\delta} / 2)$.

6. $\quad$ Draw $\boldsymbol{\mu}_{n}^{(g)} \mid \boldsymbol{Y}, \boldsymbol{s}_{n}^{(g)}, \sigma_{n}^{2,(g)}, \phi_{n}^{(g-1)}, \rho^{(g-1)}(n=1, \ldots, N)$ from $\mathrm{N}\left(\overline{\boldsymbol{m}}_{\mu, n}, \overline{\boldsymbol{M}}_{\mu, n}\right)$.

7. Draw $\phi_{n}^{(g)} \mid \boldsymbol{Y}, \boldsymbol{s}_{n}^{(g)}, \sigma_{n}^{2,(g)}, \boldsymbol{\mu}_{n}^{(g)}, \rho^{(g-1)}(n=1, \ldots, N)$ from $\mathrm{N}\left(\bar{m}_{\phi, n}, \bar{M}_{\phi, n}\right)$.

8. Draw $\rho^{(g)} \mid \boldsymbol{Y}, \boldsymbol{S}^{(g)}, \boldsymbol{\Omega}_{n}^{(g)}, \boldsymbol{\mu}_{0}^{(g)}, \boldsymbol{\mu}_{1}^{(g)}, \boldsymbol{\Phi}^{(g)}$ based on the MH algorithm.

(a) Draw $\rho^{\prime}$ from a truncated normal distribution $\operatorname{TN}_{\left(1 / \omega_{\min }, 1\right)}\left(\rho^{(g-1)}, 1\right)$ as a proposal distribution $q(\cdot)$.

(b) Calculate the acceptance probability.

$$
\alpha\left(\rho^{(g-1)}, \rho^{\prime}\right)=\min \left[\frac{\pi\left(\rho^{\prime} \mid \boldsymbol{Y}, \boldsymbol{S}^{(g)}, \boldsymbol{\Omega}_{n}^{(g)}, \boldsymbol{\mu}_{0}^{(g)}, \boldsymbol{\mu}_{1}^{(g)}, \boldsymbol{\Phi}^{(g)}\right) q\left(\rho^{\prime}, \rho^{(g-1)}\right)}{\pi\left(\rho^{(g-1)} \mid \boldsymbol{Y}, \boldsymbol{S}^{(g)}, \boldsymbol{\Omega}_{n}^{(g)}, \boldsymbol{\mu}_{0}^{(g)}, \boldsymbol{\mu}_{1}^{(g)}, \boldsymbol{\Phi}^{(g)}\right) q\left(\rho^{(g-1)}, \rho^{\prime}\right)}, 1\right] .
$$

(c) Generate $u \sim \mathrm{U}(0,1)$ and determine $\rho^{(g)}$ by using the following rule:

$$
\rho^{(g)}= \begin{cases}\rho^{\prime}, & \text { if } u \leq \alpha\left(\rho^{(g-1)}, \rho^{\prime}\right), \\ \rho^{(g-1)}, & \text { otherwise. }\end{cases}
$$

(d) Repeat steps (a)-(c) $H$ times $\left(H=10\right.$ in this study) and determine $\rho^{(g)}$.

9. Repeat steps $2-8$.

In the above algorithm, superscript $(g)$ refers to the sample from the posterior distributions obtained in the $g$ th iteration. Hyperparameters and initial parameter values are shown in Table 1. For the MH algorithm of parameter $\rho$, we use a truncated normal distribution $\operatorname{TN}_{\left(1 / \omega_{\min }, 1\right)}\left(\rho^{(g-1)}, 1\right)$ as a proposal distribution. To avoid high

${ }^{9}$ Our sampling method is also called Metropolis within Gibbs sampling, which indicates a hybrid sampler of the MH algorithm and the Gibbs sampling. We assume that the parameters are drawn from the Gibbs sampling in steps 2-7 and from the MH algorithm in step 8. However, consistent with Chib (2001, p. 3591), we use the notation of a multiple-block MH sampling because the Gibbs sampling is a special case of the multiple-block MH sampling.

${ }^{10}$ See Appendix B for more details. In the process of the multi-move Gibbs sampling, it is also necessary to apply the Hamilton filter. See Appendix C for details of the Hamilton filter. 
autocorrelation and poor mixing in the $\mathrm{MH}$ algorithm, drawing $\rho$ from the posterior distributions is repeated $H$ times within step 8. See Appendix A for more technical details. In the simulation of the posterior distributions, we discard the first 2,000 draws as a burn-in period. Descriptive statistics concerning the sampled posterior distributions are based on an additional 10,000 draws.

[Table 1]

\section{Data}

\subsection{Quarterly Indicator of State Economic Activity}

In this study, we use seasonally adjusted quarterly data of economic activity by state in Mexico. The National Institute of Statistics and Geography (Instituto Nacional de Estadistica y Geografia, INEGI) provides the Quarterly Indicator of State Economic Activity (Indicador Trimestral de la Actividad Económica Estatal, ITAEE) on their website. ${ }^{11}$ The period covered in this study is 2003:Q1-2015:Q4 for all 31 states and the Federal District (Distrito Federal). ${ }^{12}$ Although the ITAEE provides only data on aggregated sectors (primary, secondary, and tertiary; financial intermediation services indirectly measured; and total economic activity), it offers more disaggregated time-series data on state economic activity. Regarding the estimation of business cycles, the ITAEE is the best proxy for gross state product (GSP) and hence can be used to capture regional business cycles. We therefore use quarterly growth rates of total economic activity from ITAEE. The line plot of the time-series data is shown in Supplementary Information.

\subsection{Spatial Weight Matrix}

To estimate a Markov switching model with the spatial lag, we need to specify the SWM in advance. In this study, we use the distance-based SWM, which takes the following form:

$$
w_{n m}=\frac{d_{n m}^{-\eta}}{\sum_{m=1}^{N} d_{n m}^{-\eta}},
$$

where $w_{n m}$ is the $n m$ th element of the SWM, $d_{n m}$ is a bilateral distance between states $n$ and $m, N$ is the number of states, and $\eta$ is a distance decay parameter. This study uses $\eta=4$ as a baseline. ${ }^{13}$ Note that the SWM describes how a spatial spillover spreads across regions.

\footnotetext{
${ }^{11}$ Owyang et al. (2008) and Hamilton and Owyang (2012) used employment data because of data limitations. In the Mexican context, quarterly data of state economic activity are readily available and serve as a more appropriate measure than employment data in the formal sector.

${ }^{12}$ The Federal District became Mexico City (Ciudad de México) on January 29, 2016. In this study, we use Federal District because our data cover the period before the reform.

${ }^{13}$ The estimate of $\rho$ obtained from this value was close to the average estimate of $\rho$ obtained among $\eta=\{2, \ldots, 8\}$. In addition, we prefer the distance-based SWM to the contiguity-based one because the former can account for continuous space across regions.
} 
The bilateral distance is based on the route distance between two state capitals, which is taken from the Point to Point Routes (Rutas punto a punto) provided by the Ministry of Communications and Transportation (Secretaría de Comunicaciones y Transportes) of Mexico.

\section{Estimation Results}

\subsection{Determination of Recession Phase}

Figure 1 shows the probabilities of recession estimated from the Markov switching model for selective states that were in recession during the Great Recession. We define that a state is in recession at date $t$ if the probability of recession during the quarterly periods $t-1$ to $t$ takes a value of 0.5 or higher. ${ }^{14}$ The national recession period recorded by the INEGI was 2008:Apr-2009:Jun, and these six states (Aguascalientes, Baja California, Guanajuato, México, Nuevo León, and Tamaulipas) showed higher probabilities of recession than 0.5 for at least three quarterly periods from 2008:Q3 (see Supplementary Information for the results for all states). ${ }^{15}$

Following Owyang et al. (2005), in Table 2 we show when each state was in recession during the period 2003:Q12011:Q4. The black bars represent state recessions, and the recessions that occurred in 21 of the states coincided with the national recession period by the INEGI. ${ }^{16}$ We also find that some states were not in recession during the national recession period. According to our estimation results, 11 states (Baja California Sur, Colima, Chiapas, Michoacán, Morelos, Nayarit, Oaxaca, Sinaloa, Tabasco, Tlaxcala, and Zacatecas) did not experience any recessions during the study period.

[Table 2; Figure 1]

Table 3 shows that the average growth rates controlled by the spatial lag in the recession and expansion phases $\left(\mu_{0}\right.$ and $\mu_{1}$ ) differ considerably among states. ${ }^{17}$ Although all states showed that the average growth rates in the recession phase are negative, the $95 \%$ credible interval includes zero except for two states, Campeche and México. All states except Campeche show positive average growth rates, and the 95\% credible interval does not include zero for most states.

The estimated coefficient of the temporal lag is negative for most states, but only six states (Guerrero, Morelos, Oaxaca, Sinaloa, Tlaxcala, and Veracruz) have a 95\% credible interval that does not include zero, suggesting that these

\footnotetext{
${ }^{14}$ The probability of recession is calculated by $1-G^{-1} \sum_{g=1}^{G} S_{t, n}^{(g)}$, where $G$ is the number of iterations, and the superscript ( $g$ ) is the $g$ th iteration. Note that our results might not identify state recessions in their entirety. Determining whether states are in recession simply depends on whether the probability of recession is higher than 0.5 or not.

${ }^{15}$ Another national recession period is 2000:Aug-2003:Sep. However, we were not able to identify state recessions for that period because of data limitations.

${ }^{16}$ Campeche showed a different trend from the other states. Annual growth rates of real GSP $(2013=100)$ were highly negative, such as $-1.98 \%$ in $2004-2005,-2.34 \%$ in $2005-2006,-6.58 \%$ in $2006-2007,-8.48 \%$ in $2007-2008,-9.97 \%$ in $2008-2009$, $-3.43 \%$ in 2009-2010, and $-3.64 \%$ in 2010-2011. This tendency is consistent with our estimation results in Table 2.

${ }^{17}$ The complete estimation results are available in Supplementary Information.
} 
six states show countercyclical patterns. In fact, these states, except Veracruz, were estimated not to be in recession during the Great Recession.

To explain which states were likely to be in recession during the Great Recession, we additionally ran simple linear regressions for industrial structure differences across states. The explanatory variable is the intrastate industrial specialization index, which is here defined as the ratio of the GSP share of sector $s$ in state $n$ to the national share of sector $s$ at time $t$. The value 1 of this specialization index means that the share of sector $s$ in state $n$ is the same level as the national share of that sector. To avoid endogeneity, we followed the methodology adopted by Owyang et al. (2008), and thus we used the average values in the initial period (i.e., average values in the period 2003-2005) as an explanatory variable.

Table 4 shows the estimation results of these regressions. ${ }^{18}$ First, the dependent variable is the number of recessions in 2008:Q2-2009:Q2 (i.e., number of black bars in Table 2). The regression results show that states with a higher manufacturing share were likely to be in recession. The adjusted R-squared is also high $\left(R^{2}=0.273\right)$. The recession period extends by 1.6 quarters if the specialization index is two (i.e., twice as high as the share of the manufacturing sector at the national level). We also find that states with larger shares for the primary sector, mining, and educational services, health care and social assistance were likely not to be in recession even during the Great Recession. These findings are similar to those of Erquizio-Espinal (2010) and Mejía-Reyes and Erquizio-Espinal (2012), who constructed a recession resistance index by state. They found that the states of Chiapas, Oaxaca, Sinaloa, Tabasco, and Zacatecas demonstrated a relatively high level of endurance against recession. Regressions for other sectors showed no relationship and the adjusted R-squared was low.

In the second and third regressions of Table 4 , the average growth rates in the recession and expansion phases $\left(\mu_{0}\right.$ and $\mu_{1}$ ) were regressed on the specialization index of each industry separately. We find that the manufacturing sector significantly shows a lower average growth rate in the recession phase at the $1 \%$ level. The adjusted R-squared is also high $\left(R^{2}=0.407\right)$. The average growth rate in the recession phase decreases by 0.71 percentage points if the specialization index is two (i.e., twice as high as the share of the manufacturing sector at the national level). As discussed in Erquizio-Espinal (2010) and Mejía-Reyes and Erquizio-Espinal (2012), although the border states have been growing rapidly by exporting manufactured goods, their economies have been more adversely affected in the recession phase. Mejía-Reyes and Erquizio-Espinal (2012) emphasized this point as the "heads and tails" of globalization. Our results confirm that states with a higher manufacturing share are likely to experience negative economic shocks.

We also find that the average growth rate in recessions is positively correlated with the shares of the primary sector, construction, educational services, health care and social assistance, arts, entertainment and recreation, and accommodation and food services. In fact, states with higher shares of these industries were not in recession.

In the expansion phase, the results should be interpreted carefully because the regressions did not provide a good fit (i.e., the adjusted R-squared was low for all regressions). States with higher shares of wholesale and retail trades experienced higher growth rates, whereas states with a higher share of transportation and warehousing experienced relatively low growth rates in the expansion phase.

\footnotetext{
${ }^{18}$ The constant term is suppressed because the sum of shares equals 1. Campeche, Quintana Roo, and Tabasco are excluded as outliers because in these states, the mining and commerce, restaurant, and hotel sectors are comparatively large.
} 
[Tables 3-4]

\subsection{Spatial Dependence in Business Cycles}

Figure 2 shows how the recession of 2008-2009 spread across states. As we have shown that interstate business cycles are spatially dependent, Figure 2 also suggests that spatial proximity affects the propagation process of recessions. Thus, we observe that in 2008:Q2-Q3, seven states with a higher manufacturing share (Aguascalientes, Baja California, Coahuila, México, Guanajuato, Nuevo León, and Tamaulipas) had entered a recessionary phase. The recessions seem to propagate toward neighboring states from 2008:Q4 to 2009:Q2. To evaluate the extent to which regional recessions caused conditions in neighboring economies to deteriorate, we conduct a numerical simulation of spatial spillover effects, as discussed in Section 6.

[Figure 2]

In Table 3, we present the point and interval estimates of the parameters drawn from the posterior distributions. ${ }^{19}$ Our particular interest is in whether spatial dependence exists across regional business cycles, which is tested by the parameter $\rho$. The point estimates, namely mean and median, are 0.23 , and the interval estimate, namely the $95 \%$ credible interval, is $[0.18,0.27]$. Our estimation results, therefore, show that business cycles across Mexican states are spatially dependent.

Note that the spatial dependence in regional business cycles is estimated as a time-invariant parameter during the study period 2003:Q1-2015:Q4. However, spatial dependence might fluctuate over time. As a robustness check, we conducted additional analyses for time-varying spatial dependence based on spatial statistics and econometric approaches and found that spatial dependence was statistically significant only during the Great Recession of 20082009. However, the qualitative results in this study do not change only if the spatial dependence is observed during the Great Recession. See Appendix D for additional discussions. Considering the model specification with and without a spatial autoregressive process, the selection of the SWM is also important in terms of spatial spillover effects. See Appendix E for other estimation results.

\section{Numerical Simulations of Spatial Spillover Effects}

An advantage of the spatial autoregressive model is that it enables us to simulate spatial spillover effects. Based on the above-mentioned estimation results, we conduct a numerical simulation of spatial spillover effects. We quantify the extent to which states that experienced a transition from expansion to recession during the Great Recession caused deterioration in their neighboring states' economies.

From the Markov switching model (2), we have the following equation:

$$
\boldsymbol{y}_{t}=\left(\boldsymbol{I}_{N}-\rho \boldsymbol{W}\right)^{-1} \boldsymbol{\Phi} \boldsymbol{y}_{t-1}+\left(\boldsymbol{I}_{N}-\rho \boldsymbol{W}\right)^{-1}\left(\boldsymbol{\mu}_{0} \odot\left(\boldsymbol{\iota}_{N}-\boldsymbol{s}_{t}\right)+\boldsymbol{\mu}_{1} \odot \boldsymbol{s}_{t}\right)+\left(\boldsymbol{I}_{N}-\rho \boldsymbol{W}\right)^{-1} \boldsymbol{\varepsilon}_{t}
$$

\footnotetext{
${ }^{19}$ Estimation of the Markov switching model was conducted using Ox Professional 7.20 (Doornik and Ooms, 2006).
} 
where $\left(\boldsymbol{I}_{N}-\rho \boldsymbol{W}\right)^{-1}$ is a global spatial multiplier that generates spatial spillover effects following Anselin (2003). We should note that there are no spillover effects when $\rho=0$. Let $\Delta s_{t, n}$ denote a regime switch from $s_{t-1, n}=1$ to $s_{t, n}=0$, and $\Delta \boldsymbol{y}_{t}\left(\equiv \boldsymbol{y}_{t}-\boldsymbol{y}_{t-1}\right)$ denotes an $N \times 1$ vector of the first differences in growth rates $\boldsymbol{y}_{t}$ (i.e., percentage points). The direct impact of a switch to a recessionary regime in region $n$ can be calculated by the following:

$$
\frac{\Delta \boldsymbol{y}_{t}}{\Delta s_{t, n}}=-\left(\boldsymbol{I}_{N}-\rho^{*} \boldsymbol{W}\right)_{n}^{-1 \top} \times\left(\mu_{n, 1}^{*}-\mu_{n, 0}^{*}\right),
$$

where $\left(\boldsymbol{I}_{N}-\rho^{*} \boldsymbol{W}\right)_{n}^{-1 \mathrm{~T}}$ is the $n$th column vector of the matrix, and $\rho^{*}, \mu_{n, 0}^{*}$, and $\mu_{n, 1}^{*}$ are the posterior means. For the sake of simplicity, the spillover effects through the autoregressive term $\Delta \boldsymbol{y}_{t-1}$ are omitted in this specification. ${ }^{20}$

As a simulation exercise, we show the spillover effects of a switch from an expansionary to a recessionary regime for selective states (Aguascalientes, Baja California, Guanajuato, Mexico, and Nuevo Leon, Tamaulipas), which were in recession in the early phase (2008:Q3) among other states, as shown in Table 2. The estimation results of the other states are available in Supplementary Information.

Figure 3 visualizes the spatial spillover effects of a transition from expansion to recession. In panel (a), a switch to a recessionary regime in Aguascalientes decreases the quarterly growth rate in Zacatecas by 0.35 percentage points. According to Table 2, Zacatecas did not experience a recession in 2008-2009. However, our simulation results suggest that the recession in Aguascalientes caused a modest slowdown in the economy of Zacatecas.

Similarly, in panel (b), a switch to a recessionary regime in Baja California decreases the quarterly growth rate in Sonora by 0.25 percentage points. In panel (c), a switch to a recessionary regime in Guanajuato decreases the quarterly growth rate in Querétaro by 0.18 percentage points. In panel (d), a switch to a recessionary regime in México decreases the quarterly growth rate in the Federal District by 0.59 percentage points. In panel (e), a switch to a recessionary regime in Nuevo León decreases the quarterly growth rate in Coahuila by 0.50 percentage points. In panel (f), a switch to a recessionary regime in Tamaulipas decreases the quarterly growth rate in Nuevo León by 0.15 percentage points. Because the average growth rate in expansion phases was estimated to be around 1, states with higher manufacturing shares caused modest economic slowdowns in their nearest neighboring economy.

Table 2 presents the potential spatial spillover effects for all states. Note that spatial spillover effects do not exist for 11 states because they did not switch to a recessionary regime in our estimation results. As discussed in Section 1, the states with a higher manufacturing share in GSP, such as Aguascalientes, Coahuila, México, Nuevo León, and Puebla, were in recession and had negative impacts on their nearest neighboring states' economies when experiencing a switch from an expansionary to recessionary regime.

Our numerical simulations demonstrate that states that experienced a switch from an expansionary to recessionary regime during the Great Recession reduced the quarterly growth rate of their nearest neighboring state by an average of 0.26 percentage points and that of the second-nearest neighboring state by an average of 0.09 percentage points. It is also worth mentioning that the negative impacts of the switch to a recessionary regime on distant neighboring

\footnotetext{
${ }^{20}$ In this framework, the spillover effects are symmetric between the two regimes of economic recession and expansion. However, they could be asymmetric if the degree of spatial dependence changes between recession and expansion phases.
} 
economies was negligible.

[Figure 3; Table 5]

\section{Concluding Remarks}

The motivation for this study derived from the idea that spatial proximity — which facilitates business with neighboring economies through commuting, migration, and trade - might result in spatial similarities in regional business cycles. In such situations, region-specific recessions would affect the neighboring economies. Thus, to investigate the regional propagation process, we introduced a spatial autoregressive process into a Markov switching model. This framework enabled a numerical simulation of the spatial spillover effects. Thus, using data from Mexican states, we conducted numerical simulations to investigate how the economic crisis occurring in a Mexican state during the period 20082009 affected the neighboring states' economies.

We showed that a parameter measuring spatial dependence takes a positive value only during the Great Recession, suggesting that spillover effects existed across Mexican states in this period, and a region-specific shock thus caused deterioration in the neighboring states' economies through these effects. The numerical simulations showed that a switch from an expansionary to recessionary regime during the Great Recession decreased the quarterly growth rate of economic activity for the nearest state by an average of 0.26 percentage points. However, the spatial spillover effects had only limited impacts on the economies of distant states. As such, this study emphasizes that geographical proximity does matter in regional business cycles. Therefore, our results have important implications for policymakers. For example, if a regional economy begins experiencing an economic downturn, the nearest neighboring economies are also likely to experience modest economic slowdowns through the propagation process. Therefore, economic cooperation with neighboring state governments may be a solution for quicker recovery from a recession.

Finally, this study has some limitations. First, this study has not looked at the factors that strengthen spatial dependence in regional business cycles, such as trade, migration, and capital flows. Although states with higher manufacturing shares faced common external shocks during the Great Recession, it is important to identify how their economic slowdowns affected their neighboring states' economies through domestic economic factors (e.g., MejíaReyes et al., 2018). It is possible to replace the geography-based SWM with the economic-distance-based SWM, although the latter is no longer an exogenous variable, and the endogeneity must be controlled for. Second, this study considered time-invariant spatial dependence in a Markov switching model. However, this assumption might be too strong because dynamic changes in spatial dependence were not considered (e.g., Ductor and Leiva-Leon, 2016; MejíaReyes et al., 2018). Analyzing asymmetric spatial dependence between expansion and recession phases will shed light on how regions benefit from neighboring economies in an expansion phase. Thus, further research clarifying these should be undertaken.

\section{References}

Albert JH, Chib S (1993) Bayes inference via Gibbs sampling of autoregressive time series subject to Markov mean and variance shifts. J Business Econ Stat 11(1):1-15. https://doi.org/10.1080/07350015.1993.10509929 
Anselin L (1988) Spatial econometrics: methods and models. Kluwer Academic Press, Dordrecht. https://doi.org/10.1007/978-94-015-7799-1

Anselin L (2003) Spatial externalities, spatial multipliers, and spatial econometrics. Int Reg Sci Rev 26(2):153-166. https://doi.org/10.1177/0160017602250972

Anselin L, Bera AK (1998) Spatial dependence in linear regression models with an introduction to spatial econometrics. In: Ullah A, Giles DEA (eds) Handbook of Applied Economic Statistics. Marcel Dekkar, New York, pp 237-289.

Burns AF, Mitchell WC (1946) Measuring business cycles. National Bureau of Economic Research, New York.

Chib S (1995) Marginal likelihood from the Gibbs output. J Amer Stat Ass 90(432):1313-1321.

https://doi.org/10.1080/01621459.1995.10476635

Chib S (1996) Calculating posterior distributions and modal estimates in Markov mixture models. J Econom 75(1):79-97. https://doi.org/10.1016/0304-4076(95)01770-4

Chib S (2001) Markov chain Monte Carlo methods: computation and inference. In: Heckman JJ, Leamer EE (eds) Handbook of Econometrics, vol. 5. Elsevier, Amsterdam, pp 3569-3649. https://doi.org/10.1016/S15734412(01)05010-3

Chib S and Jeliazkov I (2001) Marginal likelihood from the Metropolis-Hastings output. J Amer Stat Ass 96(453):270-281. https://doi.org/10.1198/016214501750332848

Chiquiar D and Ramos-Francia M (2005) Trade and business-cycle synchronization: evidence from Mexican and U.S. manufacturing industries. N Amer J Econ Finance 16(2):187-216. https://doi.org/10.1016/j.najef.2004.12.001

Delajara M (2011) Comovimiento regional del empleo durante el ciclo económico en México. El Trimestre Económico 78(311):613-642. https://doi.org/10.20430/ete.v78i311.44

Doornik JA, Ooms M (2006) Introduction to Ox. Timberlake Consultants Press, London. http://www.doornik.com/. Accessed 29 May 2021

Ductor L, Leiva-Leon D (2016) Dynamics of global business cycle interdependence. J Int Econ 102:110-127. https://doi.org/10.1016/j.jinteco.2016.07.003 
Erquizio-Espinal A (2010) Gran recesión 2008-2009 en EE.UU. y México: un enfoque regional. Paradigma económico 2(2):5-40.

Hamilton JD (1989) A new approach to the economic analysis of nonstationary time series and the business cycle. Econometrica 57(2):357-384. https://doi.org/10.2307/1912559

Hamilton JD (2008) Regime switching models. In: Durlauf SN, Blume LE (eds) The New Palgrave Dictionary of Economics, vol. 7, 2nd edn. Palgrave Macmillan, New York, pp 53-57. https://doi.org/10.1057/978-1-349-951215_2459-1

Hamilton JD and Owyang MT (2012) The propagation of regional recessions. Rev Econ Stat 94(4):935-947. https://doi.org/10.1162/REST_a_00197

Hirata H, Kose MA, Otrok C (2013) Regionalization vs. globalization. IMF Working Papers 13/19.

Holloway G, Shankar B, Rahman S (2002) Bayesian spatial probit estimation: a primer and an application to HYV rice adoption. Agr Econ 27(3):383-402. https://doi.org/10.1016/S0169-5150(02)00070-1

Kim CJ, Nelson CR (1998) Business cycle turning points, a new coincident index, and tests of duration dependence based on a dynamic factor model with regime switching. Rev Econ Stat 80(2):188-201.

https://doi.org/10.1162/003465398557447

Kim CJ, Nelson CR (1999a) Has the U.S. economy become more stable? a Bayesian approach based on a Markovswitching model of the business cycle. Rev Econ Stat 81(4):608-616. https://doi.org/10.1162/003465399558472

Kim CJ, Nelson CR (1999b) State-space models with regime switching: classical and Gibbs-sampling approaches with applications. MIT Press, Cambridge, MA.

Kose MA, Otrok C, Whiteman CH (2003) International business cycles: world, region, and country-specific factors. Amer Econ Rev 93(4):1216-1239. https://doi.org/10.1257/000282803769206278

Lee, Lung-Fei and Jihai Yu (2010) Estimation of spatial autoregressive panel data models with fixed effects. J Econom 154(2):165-185. https://doi.org/10.1016/j.jeconom.2009.08.001

LeSage JP, Pace RK (2009) Introduction to spatial econometrics. CRC Press, Boca Raton.

Mejía-Reyes P, Campos-Chávez J (2011) Are the Mexican states and the United States business cycles synchronized? evidence from the manufacturing production. economía mexicana NUEVA ÉPOCA 20(1):79-112. 
Mejía-Reyes P, Erquizio-Espinal A (2012) Expansiones y recesiones en los Estados de México. Pearson EducaciónUniversidad de Sonora, Hermosillo.

Mejía-Reyes P, Rendón-Rojas L, Vergara-González R, Aroca P (2018) International synchronization of the Mexican states business cycles: explaining factors. N Amer J Econ Finance 44:278-288, https://doi.org/10.1016/j.najef.2018.01.009

Ohtsuka Y (2010) Estimation of regional business cycle in Japan with Markov switching spatial autoregressive-AR model. J of the Japan Statistical Society (Japanese Version) 40(2):89-109. (in Japanese)

Ord K (1975) Estimation methods for models of spatial interaction. J Amer Stat Ass 70(349):120-126. https://doi.org/10.1080/01621459.1975.10480272

Owyang MT, Piger J, Wall HJ (2005) Business cycle phases in U.S. states. Rev Econ Stat 87(4):604-616. https://doi.org/10.1162/003465305775098198

Owyang MT, Piger JM, Wall HJ, Wheeler CH (2008) The economic performance of cities: a Markov-switching approach. J Urban Econ 64(3):538-550. https://doi.org/10.1016/j.jue.2008.05.006

Owyang MT, Rapach DE, Wall HJ (2009) States and the business cycle. J Urban Econ 65(2):181-194. https://doi.org/10.1016/j.jue.2008.11.001

Sosa S (2008) External shocks and business cycle fluctuations in Mexico: how important are U.S. factors? IMF Working Papers 08/100.

Stock JH, Watson MW (1989) New indexes of coincident and leading economic indicators. In: Blanchard OJ, Fischer S (eds) NBER Macroeconomics Annual 1989, vol. 4. MIT Press, Cambridge, MA, pp 351-409. https://doi.org/10.1086/654119

Torres A, Vela O (2003) Trade integration and synchronization between the business cycles of Mexico and the United States. N Amer J Econ Finance 14(3):319-342. https://doi.org/10.1016/S1062-9408(03)00025-1

World Bank (2009) World development report 2009: reshaping economic geography. World Bank, Washington, DC. 


\section{Appendix A. Drawing $\rho$ by the Metropolis-Hastings Algorithm}

We use a truncated normal distribution as a proposal distribution. When the random variable $x$ has the truncated normal distribution $\operatorname{TN}_{(a, b)}\left(\mu, \sigma^{2}\right)$, the probability density function (p.d.f.) is as follows:

$$
q(x)= \begin{cases}\frac{(1 / \sigma) \phi((x-\mu) / \sigma)}{\Phi((b-\mu) / \sigma)-\Phi((a-\mu) / \sigma)}, & \text { if } a<x<b \\ 0, & \text { otherwise }\end{cases}
$$

where $\phi(\cdot)$ and $\Phi(\cdot)$ are the p.d.f. and the cumulative distribution function (c.d.f.) of the standard normal distribution, respectively.

To avoid high autocorrelation and poor mixing, generating $\rho$ from the posterior distributions is repeated $H$ times within the $g$ th iteration. Superscript $(h)$ refers to the sample from the posterior distributions obtained in the $h$ th iteration within the $g$ th iteration as $\rho^{(g-1, h)}$. Note that the index $h$ is reset in each $g$ th iteration.

We use the probability integral transformation method for sampling from the truncated normal distribution. We set $\mu^{(g-1, h)}=\rho^{(g-1, h)}, \sigma^{2}=1, a=1 / \omega_{\min }$, and $b=1$. Following Holloway et al. (2002), we introduce a tuning parameter $c$ into the variance term, so that the acceptance rate might fall within the interval $[0.3,0.7]{ }^{21}$

For convenience of explanation, we omit the superscript $(g-1)$ as $\rho^{(h)}=\rho^{(g-1, h)}$. Note that $\rho^{(g-1)}=$ $\rho^{(g-1,0)}$ if $h=0$. When $u$ is distributed as a uniform distribution $\mathrm{U}(0,1)$, we can draw $\rho^{\prime}$ from $\operatorname{TN}_{\left(1 / \omega_{\min }, 1\right)}\left(\rho^{(h-1)}, 1\right)$ as follows:

$$
\rho^{\prime}=\rho^{(h-1)}+c \Phi^{-1}\left(\Phi\left(1 / \omega_{\min }-\rho^{(h-1)}\right)+u\left[\Phi\left(1-\rho^{(h-1)}\right)-\Phi\left(1 / \omega_{\min }-\rho^{(h-1)}\right)\right]\right) .
$$

The acceptance probability $\alpha\left(\rho^{(h-1)}, \rho^{\prime}\right)$ is calculated by:

$$
\alpha\left(\rho^{(h-1)}, \rho^{\prime}\right)=\min \left[\frac{\pi\left(\rho^{\prime} \mid \boldsymbol{Y}, \boldsymbol{S}^{(h)}, \boldsymbol{\Omega}^{(h)}, \boldsymbol{\mu}^{(h)}, \boldsymbol{\Phi}^{(h)}\right)\left(\Phi\left(1-\rho^{(h-1)}\right)-\Phi\left(1 / \omega_{\min }-\rho^{(h-1)}\right)\right)}{\pi\left(\rho^{(h-1)} \mid \boldsymbol{Y}, \boldsymbol{S}^{(h)}, \boldsymbol{\Omega}^{(h)}, \boldsymbol{\mu}^{(h)}, \boldsymbol{\Phi}^{(h)}\right)\left(\Phi\left(1-\rho^{\prime}\right)-\Phi\left(1 / \omega_{\min }-\rho^{\prime}\right)\right)}, 1\right] .
$$

where $\pi\left(\rho \mid \boldsymbol{Y}, \boldsymbol{S}^{(h)}, \boldsymbol{\Omega}^{(h)}, \boldsymbol{\mu}^{(h)}, \boldsymbol{\Phi}^{(h)}\right)$ is calculated from equation (10). Because a standard normal distribution is symmetric, $\phi\left(\rho^{\prime}, \rho^{(h-1)}\right)$ and $\phi\left(\rho^{(h-1)}, \rho^{\prime}\right)$ are offset. We repeat this step $H=10$ times in each $g$ th iteration to avoid high autocorrelation and poor mixing. Following step 8(c) in the algorithm, we judge whether $\rho^{\prime}$ is accepted or not after the $H$ iteration as follows:

\footnotetext{
${ }^{21}$ Holloway et al. (2002) originally set the interval to [0.4,0.6], and LeSage and Pace (2009, Ch. 5) adopted the same strategy. We chose a slightly wider interval of the acceptance rate. The aim of tuning the proposals is to ensure that the MH sampling moves over the entire conditional distribution. Thus, we adjust the tuning parameter $c$ in the following way. First, we set $c=1$ as an initial value. Next, the tuning parameter $c$ is adjusted by scale factor 1.01 depending on the acceptance rate $(c \times 1.01$ if the acceptance rate exceeds 0.7 , while $c / 1.01$ if the acceptance rate falls below 0.3 ).
} 


$$
\rho^{(g)}= \begin{cases}\rho^{\prime}, & \text { if } u<\alpha\left(\rho^{(g-1, H)}, \rho^{\prime}\right) \\ \rho^{(g-1)}, & \text { otherwise. }\end{cases}
$$

\section{Appendix B. Multi-Move Gibbs Sampling for $s_{n}$}

Kim and Nelson $(1998,1999$ a, b) were the first to apply multi-move Gibbs sampling to a Markov switching model. Our explanation here is based on Kim and Nelson (1999b). For convenience of explanation, we define vectors $\tilde{\boldsymbol{s}}_{n}^{t}$ and $\boldsymbol{s}_{n}^{t}$, and a matrix $\widetilde{\boldsymbol{Y}}^{t}$ using the following notation:

$$
\tilde{\boldsymbol{s}}_{n}^{t}=\left(\begin{array}{c}
s_{1, n} \\
s_{2, n} \\
\vdots \\
s_{t, n}
\end{array}\right), \quad \boldsymbol{s}_{n}^{t}=\left(\begin{array}{c}
s_{t, n} \\
s_{t+1, n} \\
\vdots \\
s_{T, n}
\end{array}\right), \quad \widetilde{\boldsymbol{Y}}^{t}=\left(\begin{array}{cccc}
y_{1, n} & y_{1,2} & \cdots & y_{1, N} \\
y_{2, n} & y_{2,2} & \cdots & y_{2, N} \\
\vdots & \vdots & \ddots & \vdots \\
y_{t, n} & y_{t, 2} & \cdots & y_{t, N}
\end{array}\right)
$$

The aim here is to obtain $p\left(\widetilde{\boldsymbol{s}}_{n}^{T} \mid \widetilde{\boldsymbol{Y}}^{T}, \boldsymbol{\theta}\right)$. This can be rewritten as follows:

$$
\begin{aligned}
p\left(\tilde{\boldsymbol{S}}_{n}^{T} \mid \widetilde{\boldsymbol{Y}}^{T}, \boldsymbol{\theta}\right) & =p\left(s_{T, n} \mid \widetilde{\boldsymbol{Y}}^{T}, \boldsymbol{\theta}\right) p\left(\tilde{\boldsymbol{\boldsymbol { S }}}_{n}^{T-1} \mid s_{T, n}, \widetilde{\boldsymbol{Y}}^{T}, \boldsymbol{\theta}\right) \\
& =p\left(s_{T, n} \mid \widetilde{\boldsymbol{Y}}^{T}, \boldsymbol{\theta}\right) \prod_{t=1}^{T-1} p\left(s_{t, n} \mid \boldsymbol{s}_{n}^{t+1}, \widetilde{\boldsymbol{Y}}^{T}, \boldsymbol{\theta}\right) .
\end{aligned}
$$

Furthermore, the second term can be expressed as follows:

$$
p\left(s_{t, n} \mid \boldsymbol{s}_{n}^{t+1}, \widetilde{\boldsymbol{Y}}^{T}, \boldsymbol{\theta}\right) \propto p\left(s_{t+1, n} \mid s_{t, n}, \boldsymbol{\theta}\right) p\left(s_{t, n} \mid \widetilde{\boldsymbol{Y}}^{t}, \boldsymbol{\theta}\right),
$$

where the first term on the RHS represents the transition probability. Incorporating the normalizing constant, we have the following probability mass function:

$$
p\left(s_{t, n}=i \mid \boldsymbol{s}_{n}^{t+1}, \widetilde{\boldsymbol{Y}}^{T}, \boldsymbol{\theta}\right)=\frac{p\left(s_{t+1, n} \mid s_{t, n}=i, \boldsymbol{\theta}\right) p\left(s_{t, n}=i \mid \widetilde{\boldsymbol{Y}}^{t}, \boldsymbol{\theta}\right)}{\sum_{j=0}^{1} p\left(s_{t+1, n} \mid s_{t, n}=j, \boldsymbol{\theta}\right) p\left(s_{t, n}=j \mid \widetilde{\boldsymbol{Y}}^{t}, \boldsymbol{\theta}\right)^{\prime}}
$$

where $p\left(s_{t, n}=i \mid \widetilde{\boldsymbol{Y}}^{t}, \boldsymbol{\theta}\right)$ is calculated using the Hamilton filter (see Appendix C for details). The calculation step for (2) can be summarized as follows: First, we draw $s_{T, n}$ conditional on $\widetilde{\boldsymbol{Y}}^{T}$ and $\boldsymbol{\theta}$; second, given $s_{T, n}$, the sampling $s_{t, n}$ for $t=T-1, \ldots, 1$ is implemented by backward recursion based on equation (B.4).

\section{Appendix C. Hamilton Filter with Spatial Lag}

Hamilton's (1989) filter is applied to calculate the conditional probabilities $p\left(s_{t, n}=i \mid \widetilde{\boldsymbol{Y}}^{t}, \boldsymbol{\theta}\right)$ for region $n$ at date $t$. Based on Chib (1996, 2001), we explain how the Hamilton filter is applied in this study. Using scalar notation, model (2) can be rewritten as follows: 


$$
y_{t, n}=\rho \sum_{m=1}^{N} w_{n m} y_{t, m}+\phi_{n} y_{t-1, n}+\mu_{n, 0}\left(1-s_{t, n}\right)+\mu_{n, 1} s_{t, n}+\varepsilon_{t, n}, \quad \varepsilon_{t, n} \sim \text { i.i. d. } \mathrm{N}\left(0, \sigma_{n}^{2}\right) \text {. }
$$

For the conditional p.d.f. $f\left(y_{t, n} \mid s_{t, n}, \boldsymbol{y}_{t,-n}, \boldsymbol{\theta}\right)$, the expected value and variance become $\mathrm{E}\left(y_{t, n} \mid s_{t, n}, \boldsymbol{y}_{t,-n}, \boldsymbol{\theta}\right)=$ $\rho \sum_{m=1}^{N} w_{n m} y_{t, m}+\phi_{n} y_{t-1, n}+\mu_{n, 0}\left(1-s_{t, n}\right)+\mu_{n, 1} s_{t, n}$ and $\operatorname{Var}\left(y_{t, n} \mid s_{t, n}, \boldsymbol{y}_{t,-n}, \boldsymbol{\theta}\right)=\sigma_{n}^{2}$, where the subscript $-n$ of $\boldsymbol{y}_{t,-n}$ indicates that the $n$th element is excluded from the vector, and for simplicity we assumed that for each region $n$, the spatial lag $\sum_{m=1}^{N} w_{n m} y_{t, m}$ is exogenously given. Therefore, the conditional p.d.f., which is used in the iteration process of the Hamilton filter, is given by the following:

$$
\begin{aligned}
& f\left(y_{t, n} \mid s_{t, n}, \boldsymbol{y}_{t,-n}, \boldsymbol{\theta}\right)= \\
& \quad \frac{1}{\sqrt{2 \pi \sigma_{n}^{2}}} \exp \left(-\frac{\left(y_{t, n}-\rho \sum_{m=1}^{N} w_{n m} y_{t, m}-\phi_{n} y_{t-1, n}-\mu_{n, 0}\left(1-s_{t, n}\right)-\mu_{n, 1} s_{t, n}\right)^{2}}{2 \sigma_{n}^{2}}\right) .
\end{aligned}
$$

The algorithm of the Hamilton filter consists of two steps: prediction and update. The conditional p.d.f. $p\left(s_{t, n}=\right.$ $\left.i \mid \widetilde{\boldsymbol{Y}}^{t}, \boldsymbol{\theta}\right)$ is obtained by forward recursion $t=1,2, \ldots, T$.

1. Prediction Step: Calculate the probability

$$
p\left(s_{t, n}=i \mid \widetilde{\boldsymbol{Y}}^{t-1}, \boldsymbol{\theta}\right)=\sum_{j=0}^{1} p\left(s_{t, n}=i \mid s_{t-1, n}=j, \boldsymbol{\theta}\right) p\left(s_{t-1, n}=j \mid \widetilde{\boldsymbol{Y}}^{t-1}, \boldsymbol{\theta}\right),
$$

where, when $t=1, p\left(s_{0, n}=i \mid \widetilde{\boldsymbol{Y}}^{0}, \boldsymbol{\theta}\right)$ is replaced by the steady-state probabilities as follows:

$$
\pi_{n, 0}=\frac{1-p_{n, 11}}{2-p_{n, 00}-p_{n, 11}} \quad \text { and } \quad \pi_{n, 1}=\frac{1-p_{n, 00}}{2-p_{n, 00}-p_{n, 11}}
$$

2. Update Step: Calculate the probability

$$
p\left(s_{t, n}=i \mid \widetilde{\boldsymbol{Y}}^{t}, \boldsymbol{\theta}\right)=\frac{f\left(y_{t, n} \mid s_{t, n}=i, \boldsymbol{y}_{t,-n}, \boldsymbol{\theta}\right) p\left(s_{t, n}=i \mid \widetilde{\boldsymbol{Y}}^{t-1}, \boldsymbol{\theta}\right)}{\sum_{j=0}^{1} f\left(y_{t, n} \mid s_{t, n}=j, \boldsymbol{y}_{t,-n}, \boldsymbol{\theta}\right) p\left(s_{t, n}=j \mid \widetilde{\boldsymbol{Y}}^{t-1}, \boldsymbol{\theta}\right)}
$$

The probabilities $p\left(s_{t, n}=i \mid \widetilde{\boldsymbol{Y}}^{t}, \boldsymbol{\theta}\right)$ are used in the multi-move Gibbs sampling. The probabilities $p\left(s_{t, n}=i \mid \widetilde{\boldsymbol{Y}}^{t-1}, \boldsymbol{\theta}\right)$ are also used for calculating the likelihood function in the model selection. 


\section{Appendix D. Robustness Check by Spatial Econometrics}

\section{D.1. Spatial Autocorrelation}

To investigate the time-varying spatial dependence in regional business cycles, this study calculates Moran's $I$ statistics across states at time $t$ as follows:

$$
I_{t}=\frac{\boldsymbol{y}_{t}^{\top} \boldsymbol{W} \boldsymbol{y}_{t}}{\boldsymbol{y}_{t}^{\top} \boldsymbol{y}_{t}}
$$

where the SWM is based on the route distance between states with the distance decay parameter $\eta=4$.

Figure D.1 shows the calculation results of Moran's $I$ in the study period. Panel (a) shows the results in each quarterly period. The red marker indicates statistical significance at the $10 \%$ level. Importantly, spatial autocorrelation is not always significant throughout the entire period. However, spatial autocorrelation occurred during the Great Recession of 2008-2009. To mitigate the fluctuations, the centered moving average of order 3 is calculated in panel (b). The degree of spatial autocorrelation increased gradually during the Great Recession of 2008-2009 and fell after the Great Recession.

Figure D.2 shows Moran's scatterplot using annually aggregated data for 2008 and 2009. In other words, the quarterly data are pooled on a yearly basis. As discussed above, the positive spatial autocorrelation is confirmed visually during the Great Recession. Although spatial autocorrelation across regional business cycles is not obvious from the data, it is confirmed as being significant during the Great Recession.

[Figures D.1 and D.2]

\section{D.2. Spatial Panel Econometrics}

The spatial autoregressive process of dependent variable $\boldsymbol{W} \boldsymbol{y}_{t}$ considers contemporaneous interdependence across regions. One may consider another possibility of a spatial autoregressive process, that is, $\boldsymbol{W} \boldsymbol{y}_{t-1}$ instead of $\boldsymbol{W} \boldsymbol{y}_{t}$. Consider a simpler version of model (2) without a temporal autoregressive process as follows:

$$
\boldsymbol{y}_{t}=\rho \boldsymbol{W} \boldsymbol{y}_{t-1}+\boldsymbol{\mu}+\boldsymbol{\varepsilon}_{t}
$$

where $\boldsymbol{\mu}=\left(\mu_{1}, \mu_{2}, \ldots, \mu_{N}\right)$ is the fixed effect of state $n$. By successive iteration, we can show that $\boldsymbol{y}_{t}=(\boldsymbol{I}-$ $\rho \boldsymbol{W})^{-1} \boldsymbol{\mu}+(\boldsymbol{I}-\rho \boldsymbol{W})^{-1} \boldsymbol{\varepsilon}_{t}$, which is equivalent to $\boldsymbol{y}_{t}=\rho \boldsymbol{W} \boldsymbol{y}_{t}+\boldsymbol{\mu}+\boldsymbol{\varepsilon}_{t}$. Therefore, note that simultaneous spatial autoregressive processes result from dynamic spatial dependence. See LeSage and Pace (2009) for a discussion on time dependence in spatial econometric models.

To control for common external shocks across the Mexican states, this study estimated a spatial panel econometric model with fixed effects (Lee and Yu, 2010): 


$$
\boldsymbol{y}_{t}=\rho \boldsymbol{W} \boldsymbol{y}_{t}+\boldsymbol{\mu}+\tau_{t}+\boldsymbol{\varepsilon}_{t}
$$

where $\boldsymbol{\mu}=\left(\mu_{1}, \mu_{2}, \ldots, \mu_{N}\right)^{\top}$ is the fixed effect of state $n, \tau_{t}$ is the fixed effect of time $t$, and SWM is based on the route distance across states with the distance decay parameter $\eta=4$. Time fixed effects aim to control for common external shocks across the county. Year and quarter and year $\times$ quarter fixed effects are included. The parameter of interest is $\rho$, which measures the spatial dependence in economic activities.

Table D.1 shows the estimation results obtained by maximum likelihood estimation. In column (1), in which time fixed effects are not controlled for, the estimate of $\rho$ is 0.196 and significantly positive throughout the entire period. When the year and quarter fixed effects are controlled for in column (2), the magnitude of spatial dependence becomes 0.169 but remains statistically significant at the $1 \%$ level. When the year $\times$ quarter fixed effects are controlled for in column (3), the magnitude of spatial dependence becomes 0.025 and is statistically insignificant.

To estimate spatial dependence in regional business cycles under control for common external shocks, the entire period is divided into three subperiods. In columns (4) and (6), the coefficient estimates of the spatial lag are insignificant and close to zero in the pre and post periods of the Great Recession. In column (5), the parameter estimate of spatial dependence is 0.184 and significantly positive at the $10 \%$ level only during the Great Recession of 2008:Q22009:Q2, suggesting that significant spatial dependence in a subperiod results in statistical significance in the entire period. Note that the split of the study period is exogenously determined within this regression, although the Markov switching model endogenously estimates expansion and recession phases by state.

Summing up, after controlling for common external shocks across the Mexican states, we confirmed significant spatial dependence in regional business cycles only during the Great Recession. Although time-invariant spatial dependence in regional business cycles was assumed in the model, time-varying spatial dependence in regional business cycles will be more precise. Therefore, the quantitative magnitude of spatial spillover effects on neighboring economies might have a wider range than that estimated, whereas the qualitative discussion about the spatial spillover effects does not change.

[Table D.1]

\section{Appendix E. Model Selection}

We use the log marginal likelihood to compare different econometric models. Chib (1995) proposed a procedure for calculating marginal likelihood under Gibbs sampling. However, in this study, a parameter measuring spatial dependence $\rho$ is drawn by the MH algorithm, and thus we employ a method proposed by Chib and Jeliazkov (2001).

The calculation of the marginal likelihood is based on the following equation:

$$
m(\boldsymbol{Y})=\frac{L(\boldsymbol{Y} \mid \boldsymbol{\theta}) \pi(\boldsymbol{\theta})}{\pi(\boldsymbol{\theta} \mid \boldsymbol{Y})}
$$

which is termed the basic marginal likelihood identity (BMI). The BMI consists of the likelihood function, prior distribution, and posterior distribution. This identity holds at any $\boldsymbol{\theta}$. In this study, the mean of the posterior distribution 
$\boldsymbol{\theta}^{*}$ is used. Thus, by taking the logarithms of the BMI and evaluating them at $\boldsymbol{\theta}^{*}$, we can calculate the log marginal likelihood estimate as follows:

$$
\log \widehat{m}(\boldsymbol{Y})=\log L\left(\boldsymbol{Y} \mid \boldsymbol{\theta}^{*}\right)+\log \pi\left(\boldsymbol{\theta}^{*}\right)-\log \hat{\pi}\left(\boldsymbol{\theta}^{*} \mid \boldsymbol{Y}\right)
$$

Based on equation (E.2), we calculate the following three terms: the likelihood function, the prior distribution, and the posterior distribution, all evaluated at $\boldsymbol{\theta}^{*}$.

The first term on the RHS of equation (E.2) is the log likelihood function. Note that the Markov switching model includes hidden variables $\left\{\boldsymbol{s}_{t}\right\}_{t=1}^{T}$. The likelihood function thus takes the following form:

$$
L\left(\boldsymbol{Y} \mid \boldsymbol{\theta}^{*}\right)=\prod_{t=1}^{T}\left[\sum_{j=0}^{1} f\left(\boldsymbol{y}_{t} \mid \boldsymbol{s}_{t}=j, \boldsymbol{\theta}^{*}\right) p\left(\boldsymbol{s}_{t}=j \mid \widetilde{\boldsymbol{Y}}^{t-1}, \boldsymbol{\theta}^{*}\right)\right] .
$$

The second term in the brackets must be calculated in advance. This term can be obtained from the prediction step in the Hamilton filter.

The second term on the RHS of equation (E.2) is the logarithm of the joint prior distribution. As we assumed an independent prior distribution across parameters and regions, the prior distribution can be obtained as follows:

$$
\pi\left(\boldsymbol{\theta}^{*}\right)=\pi\left(\rho^{*}\right)\left[\prod_{n=1}^{N} \pi\left(\sigma_{n}^{2 *}\right) \pi\left(\boldsymbol{\mu}_{n}^{*}\right) \pi\left(\phi_{n}\right) \pi\left(p_{n, 11}^{*}\right) \pi\left(p_{n, 00}^{*}\right)\right] .
$$

The third term on the RHS of equation (E.2) is the logarithm of the joint posterior distribution, which can be rewritten as follows:

$$
\begin{aligned}
\hat{\pi}\left(\boldsymbol{\theta}^{*} \mid \boldsymbol{Y}\right)=\hat{\pi}\left(\rho^{*} \mid \boldsymbol{Y}\right)\left[\prod_{n=1}^{N} \hat{\pi}\left(\sigma_{n}^{2 *} \mid \rho^{*}, \boldsymbol{Y}\right) \hat{\pi}\left(\boldsymbol{\mu}_{n}^{*} \mid \rho^{*}, \sigma_{n}^{2 *}, \boldsymbol{Y}\right) \hat{\pi}\left(\phi_{n}^{*} \mid \rho^{*}, \sigma_{n}^{2 *}, \boldsymbol{\mu}_{n}^{*}, \boldsymbol{Y}\right)\right. \\
\left.\times \hat{\pi}\left(p_{n, 11}^{*} \mid \rho^{*}, \sigma_{n}^{2 *}, \boldsymbol{\mu}_{n}^{*}, \phi_{n}^{*}, \boldsymbol{Y}\right) \hat{\pi}\left(p_{n, 00}^{*} \mid \rho^{*}, \sigma_{n}^{2 *}, \boldsymbol{\mu}_{n}^{*}, \phi_{n}^{*}, p_{n, 11}^{*}, \boldsymbol{Y}\right)\right],
\end{aligned}
$$

where

$$
\begin{gathered}
\hat{\pi}\left(\rho^{*} \mid \boldsymbol{Y}\right)=\frac{G^{-1} \sum_{g=1}^{G} \alpha\left(\rho^{(g)}, \rho^{*} \mid \boldsymbol{Y}, \boldsymbol{S}^{(g)}, \boldsymbol{\Omega}^{(g)}, \boldsymbol{\mu}^{(g)}, \boldsymbol{\Phi}^{(g)}, \boldsymbol{p}_{11}^{(g)}, \boldsymbol{p}_{00}^{(g)}\right) q\left(\rho^{(g)}, \rho^{*}\right)}{J^{-1} \sum_{k=1}^{J} \alpha\left(\rho^{*}, \rho^{(k)} \mid \boldsymbol{Y}, \boldsymbol{S}^{(k)}, \boldsymbol{\Omega}^{(k)}, \boldsymbol{\mu}^{(k)}, \boldsymbol{\Phi}^{(k)}, \boldsymbol{p}_{11}^{(k)}, \boldsymbol{p}_{, 00}^{(k)}\right)}, \\
\hat{\pi}\left(\sigma_{n}^{2 *} \mid \rho^{*}, \boldsymbol{Y}\right)=\frac{1}{J} \sum_{k=1}^{J} \pi\left(\sigma_{n}^{2 *} \mid \rho^{*}, \boldsymbol{\mu}_{n}^{(k)}, \phi_{n}^{(k)}, p_{n, 11}^{(k)}, p_{n, 00}^{(k)}, \boldsymbol{s}_{n}^{(k)}, \boldsymbol{Y}\right), \\
\hat{\pi}\left(\boldsymbol{\mu}_{n}^{*} \mid \rho^{*}, \sigma_{n}^{2 *}, \boldsymbol{Y}\right)=\frac{1}{J} \sum_{k=1}^{J} \pi\left(\boldsymbol{\mu}_{n}^{*} \mid \rho^{*}, \sigma_{n}^{2 *}, \phi_{n}^{(k)}, p_{n, 11}^{(k)}, p_{n, 00}^{(k)}, \boldsymbol{s}_{n}^{(k)}, \boldsymbol{Y}\right), \\
\hat{\pi}\left(\phi_{n}^{*} \mid \rho^{*}, \sigma_{n}^{2 *}, \boldsymbol{\mu}_{n}^{*}, \boldsymbol{Y}\right)=\frac{1}{J} \sum_{k=1}^{J} \pi\left(\boldsymbol{\mu}_{n}^{*} \mid \rho^{*}, \sigma_{n}^{2 *}, \boldsymbol{\mu}_{n}^{*}, p_{n, 11}^{(k)}, p_{n, 00}^{(k)}, \boldsymbol{s}_{n}^{(k)}, \boldsymbol{Y}\right),
\end{gathered}
$$




$$
\begin{gathered}
\hat{\pi}\left(p_{n, 11}^{*} \mid \rho^{*}, \sigma_{n}^{2 *}, \boldsymbol{\mu}_{n}^{*}, \phi_{n}^{*}, \boldsymbol{Y}\right)=\frac{1}{J} \sum_{k=1}^{J} \pi\left(p_{n, 11}^{*} \mid \rho^{*}, \sigma_{n}^{2 *}, \boldsymbol{\mu}_{n}^{*}, \phi_{n}^{*}, p_{n, 00}^{(k)}, \boldsymbol{s}_{n}^{(k)}, \boldsymbol{Y}\right), \\
\hat{\pi}\left(p_{n, 00}^{*} \mid \rho^{*}, \sigma_{n}^{2 *}, \boldsymbol{\mu}_{n}^{*}, \phi_{n}^{*}, p_{n, 11}^{*}, \boldsymbol{Y}\right)=\frac{1}{J} \sum_{k=1}^{J} \pi\left(p_{n, 00}^{*} \mid \rho^{*}, \sigma_{n}^{2 *}, \boldsymbol{\mu}_{n}^{*}, \phi_{n}^{*}, p_{n, 11}^{*}, \boldsymbol{s}_{n}^{(k)}, \boldsymbol{Y}\right) .
\end{gathered}
$$

The superscript $(g)$ refers to the sample from the posterior distribution in the $g$ th iteration and $(k)$ refers to the sample from the reduced Gibbs runs obtained in the $k$ th iteration. Note that some of the parameters are given as a mean in the reduced Gibbs runs, and that $\rho^{(k)}$ is drawn from a proposal distribution $q\left(\rho^{*}, \rho^{(k)}\right)$. Besides the $G$ iterations, we need to implement an additional $5 \times J$ iterations for the reduced Gibbs runs. The first reduced run is for the denominator of $\hat{\pi}\left(\rho^{*} \mid \boldsymbol{Y}\right)$ and $\hat{\pi}\left(\sigma_{n}^{2 *} \mid \rho^{*}, \boldsymbol{Y}\right)$; the second is for $\hat{\pi}\left(\boldsymbol{\mu}_{n}^{*} \mid \rho^{*}, \sigma_{n}^{2 *}, \boldsymbol{Y}\right)$; the third is for $\hat{\pi}\left(\phi_{n}^{*} \mid \rho^{*}, \sigma_{n}^{2 *}, \boldsymbol{\mu}_{n}^{*}, \boldsymbol{Y}\right)$; the fourth is for $\hat{\pi}\left(p_{n, 11}^{*} \mid \rho^{*}, \sigma_{n}^{2 *}, \boldsymbol{\mu}_{n}^{*}, \phi_{n}^{*}, \boldsymbol{Y}\right)$; and the fifth is for $\hat{\pi}\left(p_{n, 00}^{*} \mid \rho^{*}, \sigma_{n}^{2 *}, \boldsymbol{\mu}_{n}^{*}, \phi_{n}^{*}, p_{n, 11}^{*}, \boldsymbol{Y}\right)$. We set $J$ to have the same number of iterations as $G$. Moreover, the numerical standard errors of the marginal likelihood estimates are also calculated. For calculation of the numerical standard errors, we need to select a lag at which the autocorrelation is small enough to be neglected. Thus, we set the lag length equal to 40 . See Chib and Jeliazkov (2001) for more details.

Table E.1 presents the log marginal likelihood estimates with numerical standard errors using the different econometric models. First, it is useful to compare estimates of the log marginal likelihood between the Markov switching model with a spatial autoregressive process (MS-SAR) and the Markov switching (MS) model because MS is a spatial case of MS-SAR when $\rho=0$. Consequently, it is supported to take into account spatial dependence in regional business cycles. Our estimation results also indicate that the Markov switching model with a first-order autoregressive process MS-AR(1) fits the data almost as well as MS-SAR. The Markov switching model with a spatial autoregressive process and a first-order autoregressive process MS-SAR-AR(1) is supported against MS-AR(1) or MS-SAR. See Supplementary Information for the full estimation results of MS, MS-AR(1), MS-SAR, and MS-SAR$\operatorname{AR}(1)$

[Table E.1]

\section{Appendix F. Map of Mexico}

State codes and names appear in Figure F.1.

[Figure F.1] 
Table 1: Prior Distributions and Initial Values

\begin{tabular}{cccc}
\hline \hline Parameter & Prior Distribution & Hyperparameters & Initial Values \\
\hline$\sigma_{n}^{2}$ & $\mathrm{IG}(\underline{\underline{v}} / 2, \underline{\delta} / 2)$ & $\underline{v}=6 ; \underline{\delta}=0.4$ & $\sigma_{n}^{2,(0)}=1$ \\
$\left(\mu_{0 n}, \mu_{0 n}\right)^{\top}$ & $\mathrm{N}_{2}\left(\underline{\boldsymbol{m}}_{\mu}, \underline{\boldsymbol{M}}_{\mu}\right)$ & $\underline{\boldsymbol{m}}_{\mu}=(-0.5,0.5)^{\top} ; \underline{\boldsymbol{M}}_{\mu}=\mathbf{I}_{2}$ & $\left(\mu_{n 0}^{(0)}, \mu_{n 1}^{(0)}\right)^{\top}=(-0.5,0.5)^{\top}$ \\
$\phi_{0 n}$ & $\mathrm{~N}_{1}\left(\underline{\underline{m}}_{\phi}, \underline{M}_{\phi}\right)$ & $\underline{m}_{\phi}=0 ; \underline{M}_{\phi}=1$ & $\phi_{0 n}^{(0)}=0$ \\
$p_{n, 00}$ & $\operatorname{Beta}\left(\underline{\alpha}_{00}, \underline{\alpha}_{01}\right)$ & $\underline{\alpha}_{00}=8 ; \underline{\alpha}_{01}=2$ & $p_{n, 00}^{(0)}=0.8$ \\
$p_{n, 11}$ & $\operatorname{Beta}\left(\underline{\alpha}_{11}, \underline{\alpha}_{10}\right)$ & $\underline{\alpha}_{11}=9 ; \underline{\alpha}_{10}=1$ & $p_{n, 11}^{(0)}=0.8$ \\
$\rho$ & $\mathrm{U}\left(1 / \omega_{\min }, 1\right)$ & & $\rho^{(0)}=0$ \\
\hline
\end{tabular}

Notes: $n=1,2, \ldots, N$. IG indicates an inverse gamma distribution. $\mathrm{N}_{1}$ and $\mathrm{N}_{2}$ indicate univariate and bivariate normal distributions, respectively. Beta indicates a beta distribution. $\mathrm{U}$ indicates a uniform distribution. 
Table 2: State Recessions

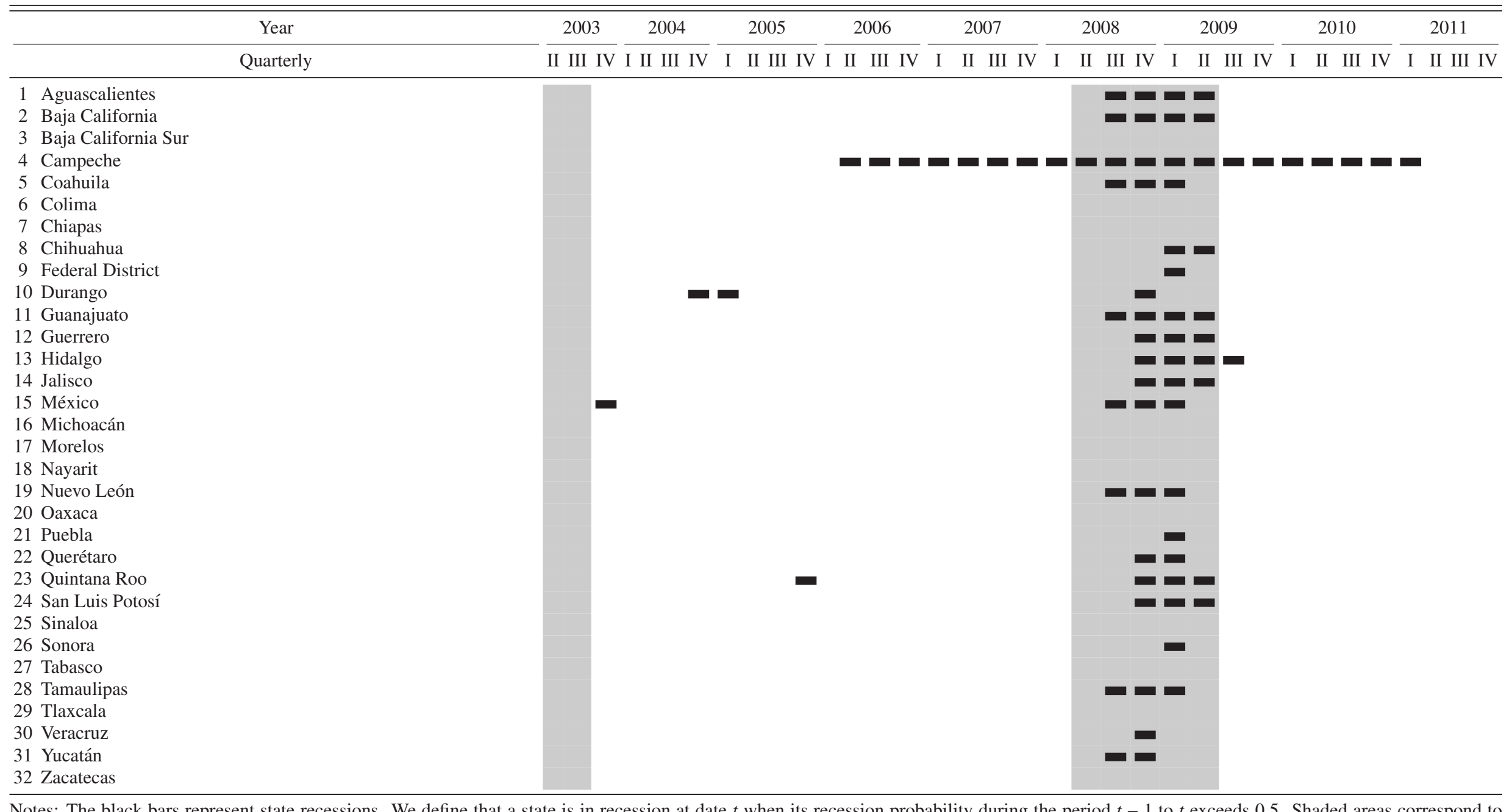

Notes: The black bars represent state recessions. We define that a state is in recession at date $t$ when its recession probability during the period $t-1$ to $t$ exceeds 0.5 . Shaded areas correspond to the dates of recessions recorded by the National Institute of Statistics and Geography (Instituto Nacional de Estadística y Geografía, INEGI). Estimation results for the period 2012:Q1-2015:Q4 are omitted. 
Table 3: Estimated Parameters from the Bayesian Inference

\begin{tabular}{|c|c|c|c|c|c|c|c|c|c|c|}
\hline & & \multicolumn{9}{|c|}{$\rho$} \\
\hline & & \multicolumn{3}{|c|}{ Mean } & \multicolumn{3}{|c|}{ Median } & \multicolumn{3}{|c|}{$95 \% \mathrm{CI}$} \\
\hline \multicolumn{2}{|r|}{ Spatial Dependence } & \multicolumn{3}{|c|}{0.23} & \multicolumn{3}{|c|}{0.23} & \multicolumn{3}{|c|}{$[0.18,0.27]$} \\
\hline & & \multicolumn{3}{|c|}{$\mu_{0}$} & \multicolumn{3}{|c|}{$\mu_{1}$} & \multicolumn{3}{|c|}{$\phi$} \\
\hline Code & State & Mean & Median & $95 \% \mathrm{CI}$ & Mean & Median & $95 \% \mathrm{CI}$ & Mean & Median & $95 \% \mathrm{CI}$ \\
\hline 1 & Aguascalientes & -0.83 & -0.81 & {$[-2.83,0.84]$} & 1.21 & 1.22 & {$[0.45,2.00]$} & -0.13 & -0.12 & {$[-0.40,0.15]$} \\
\hline 2 & Baja California & -0.84 & -0.72 & {$[-2.61,0.44]$} & 0.67 & 0.63 & {$[-0.06,1.57]$} & 0.08 & 0.09 & {$[-0.19,0.34]$} \\
\hline 3 & Baja California Sur & -0.29 & -0.20 & {$[-2.14,1.08]$} & 1.23 & 1.20 & {$[0.22,2.42]$} & -0.19 & -0.20 & {$[-0.46,0.07]$} \\
\hline 4 & Campeche & -1.55 & -1.49 & {$[-2.72,-0.70]$} & -0.27 & -0.39 & {$[-1.28,1.37]$} & -0.03 & -0.03 & {$[-0.31,0.26]$} \\
\hline 5 & Coahuila & -0.96 & -0.80 & {$[-3.08,0.41]$} & 0.61 & 0.58 & {$[-0.23,1.64]$} & 0.29 & 0.29 & {$[0.04,0.53]$} \\
\hline 6 & Colima & -0.39 & -0.28 & {$[-2.16,0.81]$} & 0.86 & 0.82 & {$[0.05,1.86]$} & -0.13 & -0.13 & {$[-0.39,0.14]$} \\
\hline 7 & Chiapas & -0.59 & -0.47 & {$[-2.12,0.36]$} & 0.42 & 0.36 & {$[-0.23,1.48]$} & -0.03 & -0.03 & {$[-0.29,0.23]$} \\
\hline 8 & Chihuahua & -2.28 & -2.38 & {$[-4.34,0.20]$} & 0.98 & 0.99 & {$[0.44,1.48]$} & -0.11 & -0.11 & {$[-0.34,0.16]$} \\
\hline 9 & Federal District & -0.72 & -0.52 & {$[-2.85,0.57]$} & 0.63 & 0.62 & {$[0.13,1.22]$} & -0.04 & -0.04 & {$[-0.32,0.22]$} \\
\hline 10 & Durango & -0.57 & -0.34 & {$[-2.45,0.49]$} & 0.63 & 0.60 & {$[0.13,1.39]$} & -0.22 & -0.22 & {$[-0.51,0.07]$} \\
\hline 11 & Guanajuato & -0.95 & -1.01 & {$[-2.37,0.54]$} & 1.00 & 1.00 & {$[0.31,1.72]$} & -0.09 & -0.09 & {$[-0.36,0.21]$} \\
\hline 12 & Guerrero & -0.51 & -0.41 & {$[-2.15,0.59]$} & 0.80 & 0.76 & {$[0.23,1.61]$} & -0.35 & -0.35 & {$[-0.61,-0.09]$} \\
\hline 13 & Hidalgo & -0.98 & -1.02 & {$[-2.60,0.56]$} & 0.99 & 0.99 & {$[0.38,1.61]$} & -0.07 & -0.07 & {$[-0.38,0.25]$} \\
\hline 14 & Jalisco & -1.32 & -1.40 & {$[-2.88,0.32]$} & 0.67 & 0.68 & {$[0.16,1.14]$} & -0.01 & -0.01 & {$[-0.32,0.33]$} \\
\hline 15 & México & -2.11 & -2.16 & {$[-3.27,-0.51]$} & 0.75 & 0.75 & {$[0.39,1.10]$} & 0.04 & 0.04 & {$[-0.16,0.25]$} \\
\hline 16 & Michoacán & -0.70 & -0.53 & {$[-2.68,0.59]$} & 0.66 & 0.63 & {$[-0.03,1.51]$} & -0.20 & -0.20 & {$[-0.50,0.10]$} \\
\hline 17 & Morelos & -0.57 & -0.48 & {$[-2.17,0.58]$} & 0.64 & 0.58 & {$[-0.25,1.87]$} & -0.31 & -0.31 & {$[-0.57,-0.05]$} \\
\hline 18 & Nayarit & -0.34 & -0.23 & {$[-2.09,0.85]$} & 0.98 & 0.93 & {$[0.13,2.18]$} & -0.07 & -0.08 & {$[-0.35,0.20]$} \\
\hline 19 & Nuevo León & -1.09 & -1.15 & {$[-2.65,0.58]$} & 1.02 & 1.02 & {$[0.48,1.58]$} & -0.05 & -0.05 & {$[-0.33,0.20]$} \\
\hline 20 & Oaxaca & -0.37 & -0.19 & {$[-2.34,0.78]$} & 0.82 & 0.79 & {$[0.28,1.52]$} & -0.48 & -0.48 & {$[-0.75,-0.20]$} \\
\hline 21 & Puebla & -0.72 & -0.57 & {$[-2.84,0.68]$} & 0.85 & 0.82 & {$[0.03,1.85]$} & -0.02 & -0.02 & {$[-0.33,0.28]$} \\
\hline 22 & Querétaro & -0.26 & -0.13 & {$[-1.99,0.82]$} & 1.00 & 0.96 & {$[0.37,1.80]$} & 0.16 & 0.16 & {$[-0.13,0.43]$} \\
\hline 23 & Quintana Roo & -2.06 & -2.21 & {$[-4.30,0.67]$} & 1.74 & 1.77 & {$[0.71,2.56]$} & -0.16 & -0.17 & {$[-0.40,0.11]$} \\
\hline 24 & San Luis Potosí & -1.55 & -1.66 & {$[-3.11,0.46]$} & 1.06 & 1.07 & {$[0.52,1.56]$} & -0.24 & -0.25 & {$[-0.53,0.11]$} \\
\hline 25 & Sinaloa & -0.35 & -0.24 & {$[-2.11,0.86]$} & 0.91 & 0.87 & {$[0.08,1.93]$} & -0.38 & -0.37 & {$[-0.62,-0.12]$} \\
\hline 26 & Sonora & -1.28 & -1.19 & {$[-3.75,0.66]$} & 1.02 & 1.02 & {$[0.33,1.75]$} & -0.07 & -0.07 & {$[-0.30,0.18]$} \\
\hline 27 & Tabasco & -0.23 & -0.11 & {$[-1.91,0.89]$} & 1.08 & 1.04 & {$[0.45,1.84]$} & -0.10 & -0.10 & {$[-0.38,0.17]$} \\
\hline 28 & Tamaulipas & -1.28 & -1.27 & {$[-3.20,0.35]$} & 0.60 & 0.59 & {$[0.01,1.25]$} & 0.16 & 0.16 & {$[-0.11,0.41]$} \\
\hline 29 & Tlaxcala & -0.69 & -0.63 & {$[-2.39,0.66]$} & 0.72 & 0.69 & {$[-0.43,2.10]$} & -0.41 & -0.41 & {$[-0.64,-0.16]$} \\
\hline 30 & Veracruz & -0.35 & -0.24 & {$[-2.03,0.72]$} & 0.93 & 0.87 & {$[0.22,2.01]$} & -0.44 & -0.44 & {$[-0.69,-0.20]$} \\
\hline 31 & Yucatán & -0.15 & -0.09 & {$[-1.92,1.11]$} & 1.32 & 1.30 & {$[0.76,1.97]$} & -0.28 & -0.28 & {$[-0.60,0.03]$} \\
\hline 32 & Zacatecas & -0.23 & -0.15 & {$[-1.96,0.98]$} & 1.27 & 1.19 & {$[0.41,2.66]$} & -0.24 & -0.24 & {$[-0.49,0.02]$} \\
\hline
\end{tabular}

Notes: 95\% CI indicates 95\% credible interval. 
Table 4: OLS Regression Results of Industrial Specialization Index

\begin{tabular}{|c|c|c|c|c|c|c|c|c|c|}
\hline \multirow[b]{3}{*}{ Explanatory Variables } & \multicolumn{6}{|c|}{$\begin{array}{c}\text { Dependent Variable: } \\
\text { Recession } \\
\text { Growth Rate } \mu_{0}\end{array}$} & \multirow{2}{*}{\multicolumn{3}{|c|}{$\begin{array}{c}\begin{array}{c}\text { Expansion } \\
\text { Growth Rate } \mu_{1}\end{array} \\
\text { OLS }\end{array}$}} \\
\hline & \multicolumn{3}{|c|}{ OLS } & \multicolumn{3}{|c|}{ OLS } & & & \\
\hline & Coef. & S.E. & $R^{2}$ & Coef. & S.E. & $R^{2}$ & Coef. & S.E. & $R^{2}$ \\
\hline 1. Agriculture, forestry, fishing, and hunting & $-0.739^{* * *}$ & $(0.238)$ & 0.138 & $0.183^{* *}$ & $(0.105)$ & 0.038 & -0.035 & $(0.062)$ & -0.024 \\
\hline 2. Mining & $-0.531^{* * *}$ & $(0.181)$ & 0.052 & $0.159^{* * *}$ & $(0.047)$ & 0.019 & $0.040^{*}$ & $(0.025)$ & -0.021 \\
\hline 3. Utilities & 0.163 & $(0.432)$ & -0.028 & 0.034 & $(0.101)$ & -0.034 & 0.002 & $(0.045)$ & -0.036 \\
\hline 4. Construction & $-0.700^{*}$ & $(0.503)$ & 0.022 & $0.357^{* * *}$ & $(0.148)$ & 0.067 & 0.059 & $(0.089)$ & -0.023 \\
\hline 5. Manufacturing & $1.559^{* * *}$ & $(0.435)$ & 0.273 & $-0.710^{* * *}$ & $(0.195)$ & 0.407 & -0.041 & $(0.101)$ & -0.029 \\
\hline 6. Wholesale trade; Retail trade & 1.382 & $(1.259)$ & 0.001 & -0.141 & $(0.425)$ & -0.033 & $0.328^{*}$ & $(0.236)$ & 0.027 \\
\hline 7. Transportation and warehousing & -0.323 & $(1.011)$ & -0.032 & 0.245 & $(0.376)$ & -0.020 & $-0.292^{* * *}$ & $(0.111)$ & 0.061 \\
\hline 8. Information and cultural industries & -0.013 & $(0.192)$ & -0.036 & -0.037 & $(0.081)$ & -0.034 & -0.027 & $(0.055)$ & -0.031 \\
\hline 9. Finance and insurance & 0.062 & $(0.407)$ & -0.035 & -0.033 & $(0.110)$ & -0.035 & $-0.121^{* * *}$ & $(0.046)$ & -0.001 \\
\hline 10. Real estate and rental and leasing & -0.399 & $(1.226)$ & -0.032 & -0.212 & $(0.582)$ & -0.028 & -0.220 & $(0.192)$ & 0.003 \\
\hline 11. Professional, scientific, and technical services & 0.276 & $(0.463)$ & -0.025 & -0.142 & $(0.195)$ & -0.016 & 0.041 & $(0.147)$ & -0.028 \\
\hline 12. Management of companies and enterprises & $-0.317^{*}$ & $(0.199)$ & -0.011 & 0.084 & $(0.069)$ & -0.024 & $-0.076^{* * *}$ & $(0.028)$ & 0.007 \\
\hline 13. Administrative and support, waste management, and remediation services & 0.240 & $(0.361)$ & -0.027 & 0.125 & $(0.118)$ & -0.020 & -0.020 & $(0.077)$ & -0.034 \\
\hline 14. Educational services; Health care and social assistance & $-1.627^{* *}$ & $(0.949)$ & 0.084 & $0.661^{* * *}$ & $(0.281)$ & 0.101 & -0.150 & $(0.161)$ & -0.004 \\
\hline 15. Arts, entertainment, and recreation; Accommodation and food services & -0.124 & $(0.170)$ & -0.022 & $0.143^{* * *}$ & $(0.051)$ & 0.091 & 0.022 & $(0.019)$ & -0.022 \\
\hline 16. Other services & -0.438 & $(1.125)$ & -0.030 & 0.048 & $(0.427)$ & -0.035 & -0.001 & $(0.181)$ & -0.036 \\
\hline
\end{tabular}

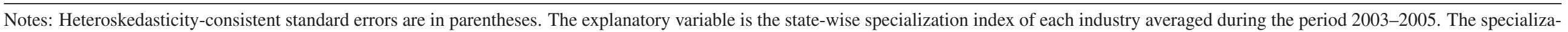

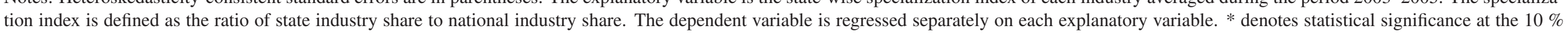

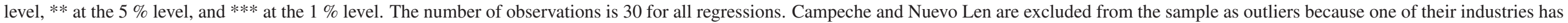
a specialization index that is 10 times higher than the average. $R^{2}$ indicates the adjusted coefficient of determination. 
Table 5: Ranking of Cumulative Spillover Effects by State

\begin{tabular}{|c|c|c|c|c|c|c|c|}
\hline \multirow[b]{2}{*}{ Code } & \multirow[b]{2}{*}{ Origin State } & \multicolumn{3}{|c|}{ First } & \multicolumn{3}{|c|}{ Second } \\
\hline & & Code & Destination State & PP & Code & Destination State & PP \\
\hline 1 & Aguascalientes* & 32 & Zacatecas & -0.35 & 11 & Guanajuato & -0.06 \\
\hline 2 & Baja California* & 26 & Sonora & -0.25 & 25 & Sinaloa & -0.03 \\
\hline 3 & Baja California Sur & 2 & Baja California & -0.16 & 26 & Sonora & -0.07 \\
\hline 4 & Campeche & 31 & Yucatán & -0.28 & 23 & Quintana Roo & -0.01 \\
\hline 5 & Coahuila* & 19 & Nuevo León & -0.37 & 32 & Zacatecas & -0.00 \\
\hline 6 & Colima & 14 & Jalisco & -0.23 & 18 & Nayarit & -0.03 \\
\hline 7 & Chiapas & 27 & Tabasco & -0.20 & 4 & Campeche & -0.01 \\
\hline 8 & Chihuahua* & 10 & Durango & -0.15 & 5 & Coahuila & -0.15 \\
\hline 9 & Distrito Federal* & 15 & México & -0.17 & 17 & Morelos & -0.06 \\
\hline 10 & Durango* & 32 & Zacatecas & -0.14 & 1 & Aguascalientes & -0.06 \\
\hline 11 & Guanajuato* & 22 & Querétaro & -0.18 & 16 & Michoacán & -0.09 \\
\hline 12 & Guerrero* & 17 & Morelos & -0.19 & 9 & Distrito Federal & -0.07 \\
\hline 13 & Hidalgo* & 9 & Distrito Federal & -0.27 & 29 & Tlaxcala & -0.10 \\
\hline 14 & Jalisco* & 6 & Colima & -0.15 & 18 & Nayarit & -0.13 \\
\hline 15 & México* & 9 & Distrito Federal & -0.59 & 17 & Morelos & -0.05 \\
\hline 16 & Michoacán & 11 & Guanajuato & -0.11 & 22 & Querétaro & -0.10 \\
\hline 17 & Morelos & 9 & Distrito Federal & -0.19 & 15 & México & -0.05 \\
\hline 18 & Nayarit & 14 & Jalisco & -0.23 & 6 & Colima & -0.03 \\
\hline 19 & Nuevo León* & 5 & Coahuila & -0.50 & 28 & Tamaulipas & -0.00 \\
\hline 20 & Oaxaca & 21 & Puebla & -0.10 & 29 & Tlaxcala & -0.08 \\
\hline 21 & Puebla & 29 & Tlaxcala & -0.36 & 9 & Distrito Federal & -0.00 \\
\hline 22 & Querétaro* & 11 & Guanajuato & -0.11 & 15 & México & -0.05 \\
\hline 23 & Quintana Roo* & 4 & Campeche & -0.49 & 31 & Yucatán & -0.45 \\
\hline 24 & San Luis Potosí* & 11 & Guanajuato & -0.18 & 32 & Zacatecas & -0.16 \\
\hline 25 & Sinaloa & 18 & Nayarit & -0.09 & 10 & Durango & -0.07 \\
\hline 26 & Sonora* & 2 & Baja California & -0.18 & 25 & Sinaloa & -0.17 \\
\hline 27 & Tabasco & 7 & Chiapas & -0.21 & 4 & Campeche & -0.05 \\
\hline 28 & Tamaulipas* & 19 & Nuevo León & -0.15 & 24 & San Luis Potosí & -0.11 \\
\hline 29 & Tlaxcala & 21 & Puebla & -0.33 & 9 & Distrito Federal & -0.00 \\
\hline 30 & Veracruz* & 21 & Puebla & -0.17 & 29 & Tlaxcala & -0.12 \\
\hline 31 & Yucatán* & 4 & Campeche & -0.33 & 23 & Quintana Roo & -0.01 \\
\hline \multirow[t]{2}{*}{32} & Zacatecas & 1 & Aguascalientes & -0.29 & 24 & San Luis Potosí & -0.04 \\
\hline & & & Average* & -0.26 & & Average* & -0.09 \\
\hline
\end{tabular}

Notes: Based on equation (21). * denotes the states that experienced a transition from expansion to recession during the Great Recession of 2008-2009. PP denotes percentage point. Average spillover effects are calculated across the 19 states that experienced a transition from expansion to recession. 
Table D.1: Maximum Likelihood Estimation Results of Spatial Panel Econometric Models

\begin{tabular}{|c|c|c|c|c|c|c|}
\hline \multirow[b]{3}{*}{ Explanatory Variable } & \multicolumn{6}{|c|}{ Dependent Variable: Growth Rate of Indicator of Economic Activity } \\
\hline & \multicolumn{3}{|c|}{ Entire Period } & \multicolumn{3}{|c|}{ Subperiod } \\
\hline & $\begin{array}{c}\text { 2003:Q1- } \\
\text { 2015:Q4 } \\
(1)\end{array}$ & $\begin{array}{c}\text { 2003:Q1- } \\
\text { 2015:Q4 } \\
\text { (2) }\end{array}$ & $\begin{array}{c}2003: \mathrm{Q} 1- \\
2015: \mathrm{Q} 4 \\
(3)\end{array}$ & $\begin{array}{c}\text { 2003:Q1- } \\
\text { 2008:Q1 } \\
(4)\end{array}$ & $\begin{array}{c}\text { 2008:Q2- } \\
\text { 2009:Q2 } \\
\quad(5)\end{array}$ & $\begin{array}{c}2009: \mathrm{Q} 3- \\
2015: \mathrm{Q} 4 \\
(6)\end{array}$ \\
\hline Spatial Lag of Dependent Variable $(\rho)$ & $\begin{array}{c}0.196^{* * *} \\
(0.024)\end{array}$ & $\begin{array}{c}0.169^{* * *} \\
(0.025)\end{array}$ & $\begin{array}{c}0.025 \\
(0.027)\end{array}$ & $\begin{array}{l}-0.017 \\
(0.045)\end{array}$ & $\begin{array}{c}0.184^{*} \\
(0.093)\end{array}$ & $\begin{array}{l}-0.007 \\
(0.039)\end{array}$ \\
\hline State Fixed Effect & Yes & Yes & Yes & Yes & Yes & Yes \\
\hline Year and Quarter Fixed Effects & No & Yes & No & No & No & No \\
\hline Year $\times$ Quarter Fixed Effects & No & No & Yes & Yes & Yes & Yes \\
\hline Number of Observations & 1632 & 1632 & 1632 & 608 & 160 & 832 \\
\hline Number of States & 32 & 32 & 32 & 32 & 32 & 32 \\
\hline
\end{tabular}

Notes: Standard errors are in parentheses. * denotes statistical significance at the $10 \%$ level, $* *$ at the $5 \%$ level, and $* * *$ at the $1 \%$ level. The spatial weight matrix is based on the route distance across states with distance decay parameter $\eta=4$. 
Table E.1: Log Marginal Likelihood Estimate

\begin{tabular}{lcccc}
\hline \hline & \multicolumn{2}{c}{ Spatial Dependence $\rho$} & \multicolumn{2}{c}{ Log Marginal Likelihood } \\
\cline { 2 - 3 } \cline { 4 - 5 } Model Fitted & Mean & $95 \%$ CI & Estimate & NSE \\
\hline MS & & & -3646.468 & $(0.868)$ \\
MS-AR(1) & & & -3557.985 & $(0.258)$ \\
MS-SAR & 0.359 & {$[0.301,0.417]$} & -3553.054 & $(2.321)$ \\
SWM: Distance $(\eta=2)$ & 0.260 & {$[0.211,0.308]$} & -3569.472 & $(0.727)$ \\
SWM: Distance $(\eta=3)$ & 0.220 & {$[0.174,0.263]$} & -3576.336 & $(0.590)$ \\
SWM: Distance $(\eta=4)$ & 0.199 & {$[0.158,0.241]$} & -3580.036 & $(1.082)$ \\
SWM: Distance $(\eta=5)$ & 0.185 & {$[0.144,0.225]$} & -3582.196 & $(2.658)$ \\
SWM: Distance $(\eta=6)$ & 0.178 & {$[0.139,0.218]$} & -3583.984 & $(0.653)$ \\
SWM: Distance $(\eta=7)$ & 0.172 & {$[0.133,0.210]$} & -3585.177 & $(0.188)$ \\
SWM: Distance $(\eta=8)$ & & & & $(1.812)$ \\
MS-SAR-AR1 & 0.372 & {$[0.313,0.432]$} & -3433.998 & $(0.670)$ \\
SWM: Distance $(\eta=2)$ & 0.267 & {$[0.217,0.317]$} & -3452.734 & $(0.333)$ \\
SWM: Distance $(\eta=3)$ & 0.224 & {$[0.179,0.269]$} & -3459.408 & $(1.177)$ \\
SWM: Distance $(\eta=4)$ & 0.200 & {$[0.160,0.242]$} & -3462.818 & $(0.835)$ \\
SWM: Distance $(\eta=5)$ & 0.188 & {$[0.147,0.229]$} & -3466.452 & $(0.496)$ \\
SWM: Distance $(\eta=6)$ & 0.179 & {$[0.140,0.219]$} & -3468.297 & $(0.444)$ \\
SWM: Distance $(\eta=7)$ & 0.173 & {$[0.134,0.212]$} & -3469.719 & \\
SWM: Distance $(\eta=8)$ & & & & \\
\hline
\end{tabular}

Notes: $G=J=10,000$. SWM indicates a spatial weight matrix. $\eta$ is a distance decay parameter. $95 \%$ CI indicates $95 \%$ credible interval. NSE indicates the numerical standard errors of the marginal likelihood estimates. Models shown in the table are as follows: MS: $\boldsymbol{y}_{t}=\boldsymbol{\mu}_{0} \odot\left(\boldsymbol{\iota}_{N}-\boldsymbol{s}_{t}\right)+\boldsymbol{\mu}_{1} \odot \boldsymbol{s}_{t}+\boldsymbol{\varepsilon}_{t}$, MS-AR(1): $\boldsymbol{y}_{t}=\boldsymbol{\Phi} \boldsymbol{y}_{t-1}+\boldsymbol{\mu}_{0} \odot\left(\boldsymbol{\iota}_{N}-\boldsymbol{s}_{t}\right)+\boldsymbol{\mu}_{1} \odot \boldsymbol{s}_{t}+\boldsymbol{\varepsilon}_{t}$, MS-SAR: $\boldsymbol{y}_{t}=\rho \boldsymbol{W} \boldsymbol{y}_{t}+\boldsymbol{\mu}_{0} \odot\left(\boldsymbol{t}_{N}-\boldsymbol{s}_{t}\right)+\boldsymbol{\mu}_{1} \odot \boldsymbol{s}_{t}+\boldsymbol{\varepsilon}_{t}, \mathrm{MS}-\mathrm{SAR}-\mathrm{AR}(1): \boldsymbol{y}_{t}=\rho \boldsymbol{W} \boldsymbol{y}_{t}+\boldsymbol{\Phi} \boldsymbol{y}_{t-1}+\boldsymbol{\mu}_{0} \odot\left(\boldsymbol{t}_{N}-\boldsymbol{s}_{t}\right)+\boldsymbol{\mu}_{1} \odot \boldsymbol{s}_{t}+\boldsymbol{\varepsilon}_{t}$ 


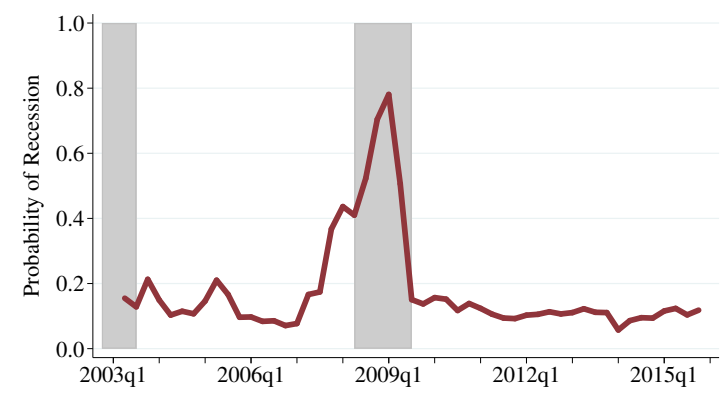

(a) Aguascalientes

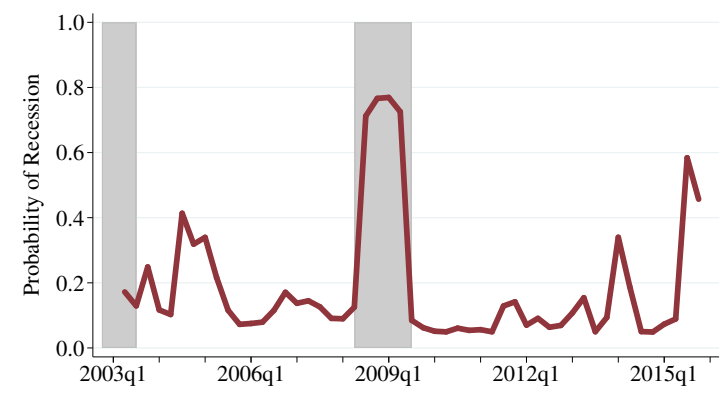

(c) Guanajuato

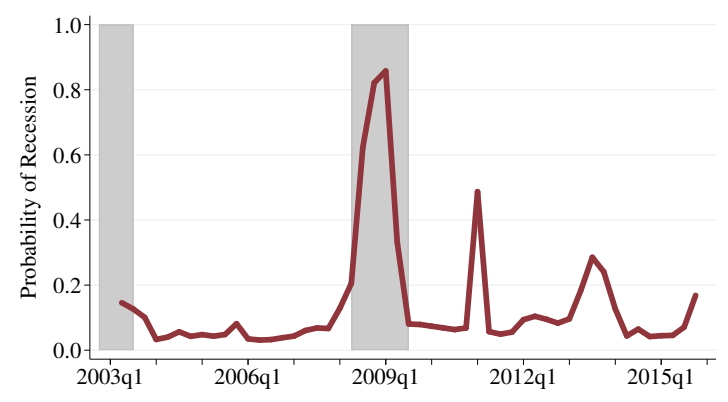

(e) Nuevo León

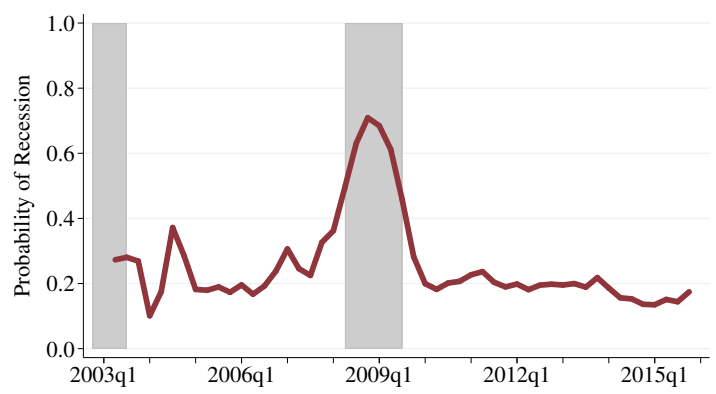

(b) Baja California

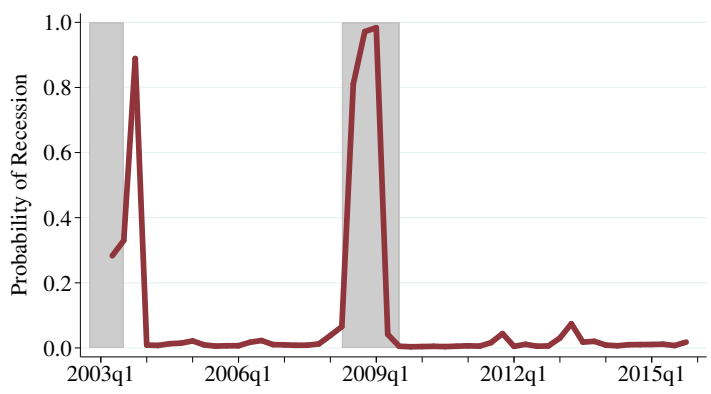

(d) Mexico

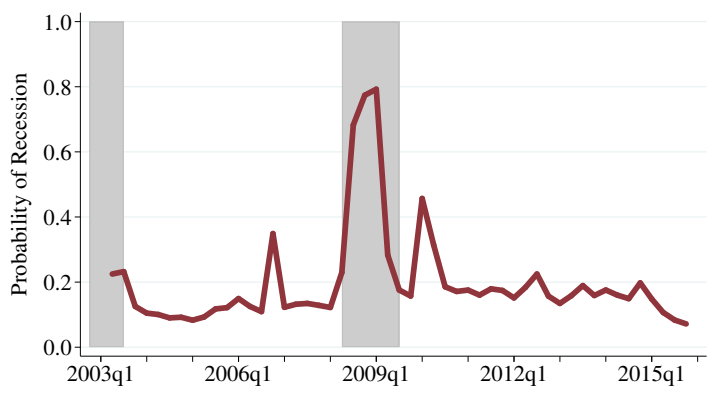

(f) Tamaulipas

Figure 1: Probabilities of Recession for Selected States

Notes: Recession probabilities estimated from MS-SAR-AR(1) are shown. Shaded areas correspond to the dates of recessions by INEGI. 


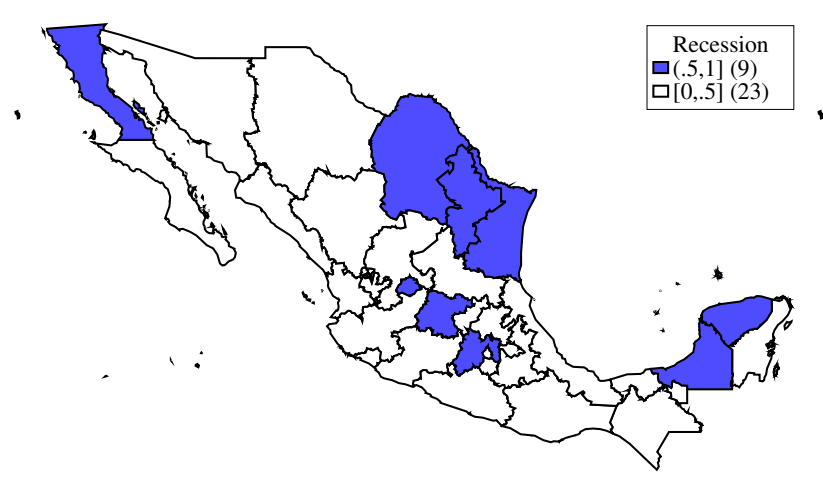

(a) 2008:Q3

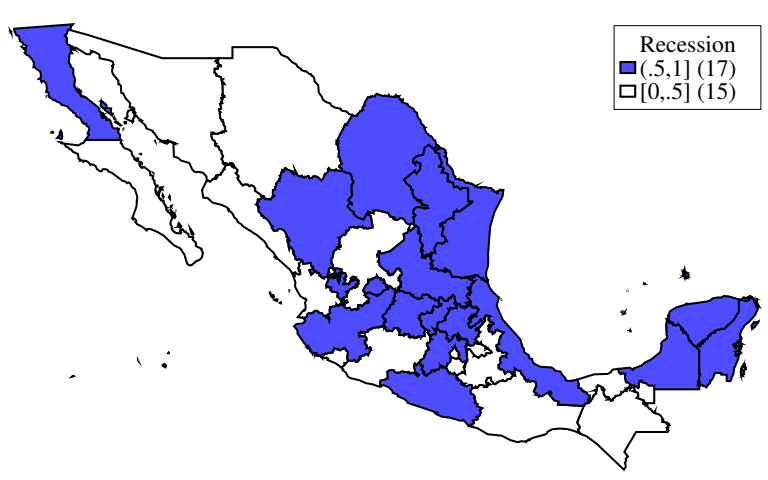

(b) $2008: \mathrm{Q} 4$

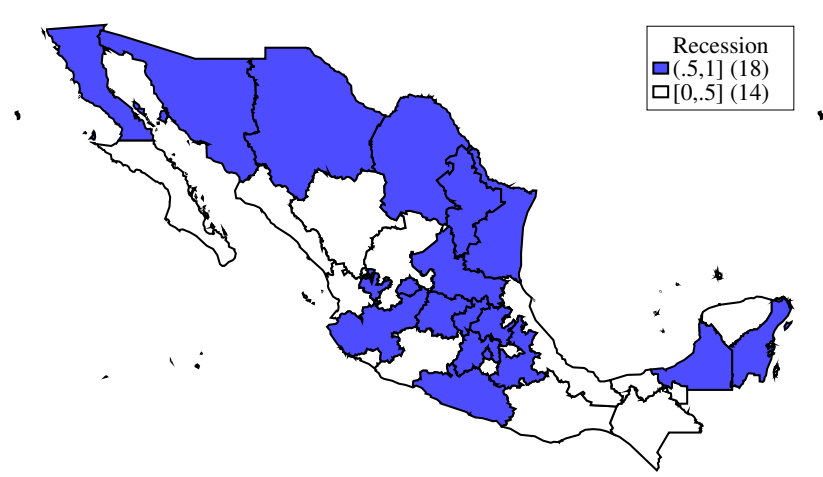

(c) $2009: \mathrm{Q} 1$

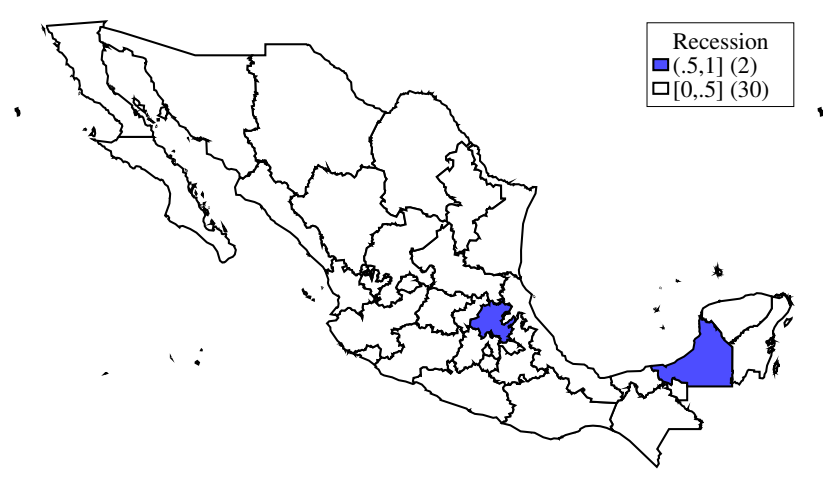

(e) $2009: \mathrm{Q} 3$

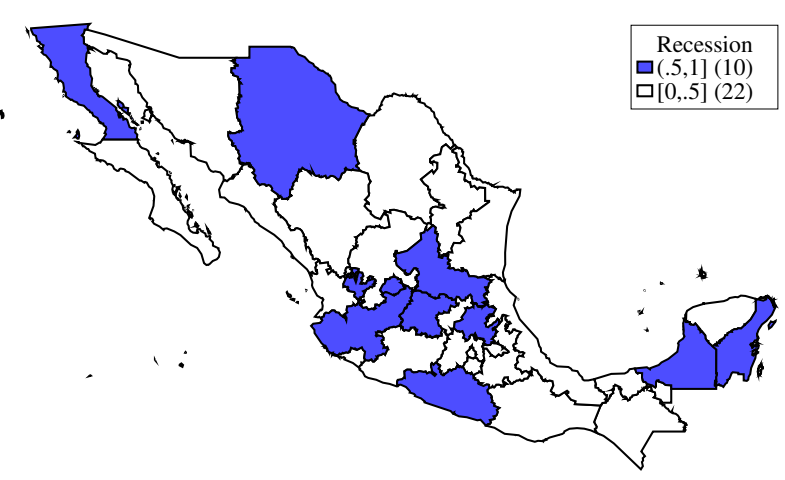

(d) 2009:Q2

Figure 2: Regional Distribution of State Recession

Notes: Colored states are in recession. 


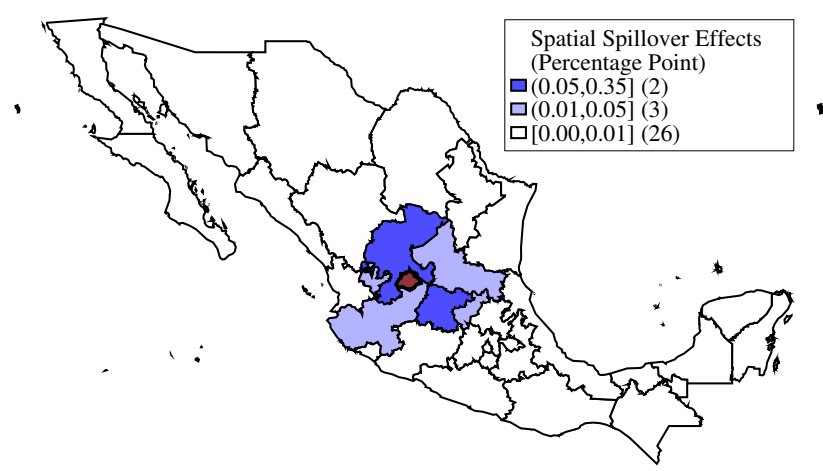

(a) Aguascalientes

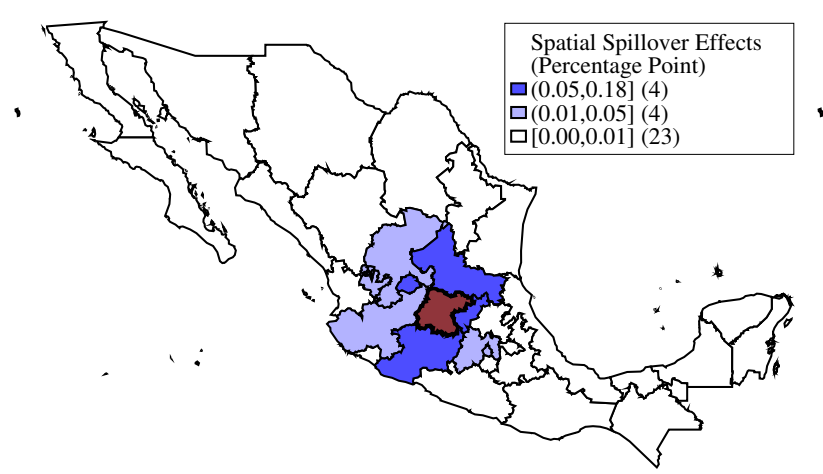

(c) Guanajuato

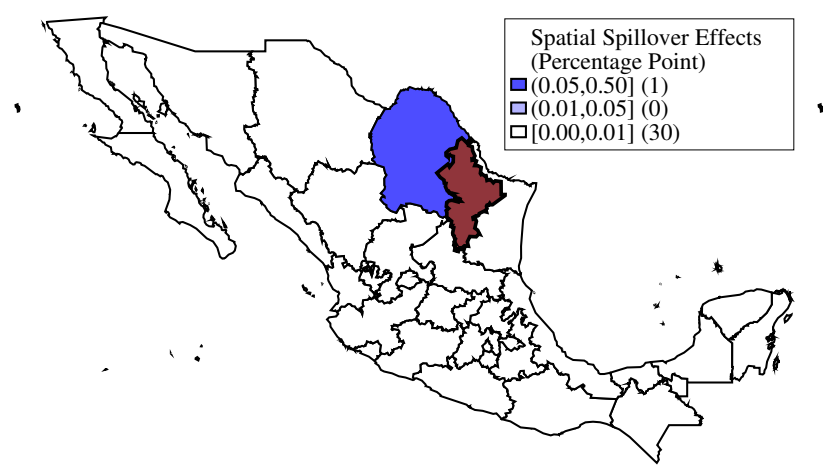

(e) Nuevo León

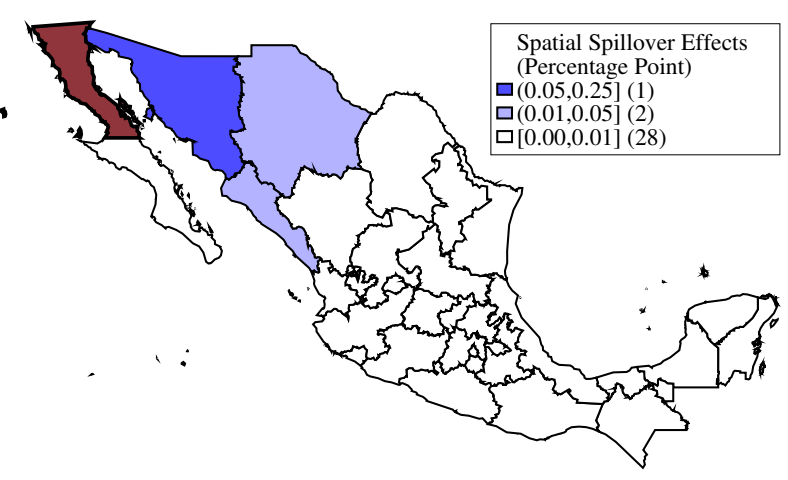

(b) Baja California

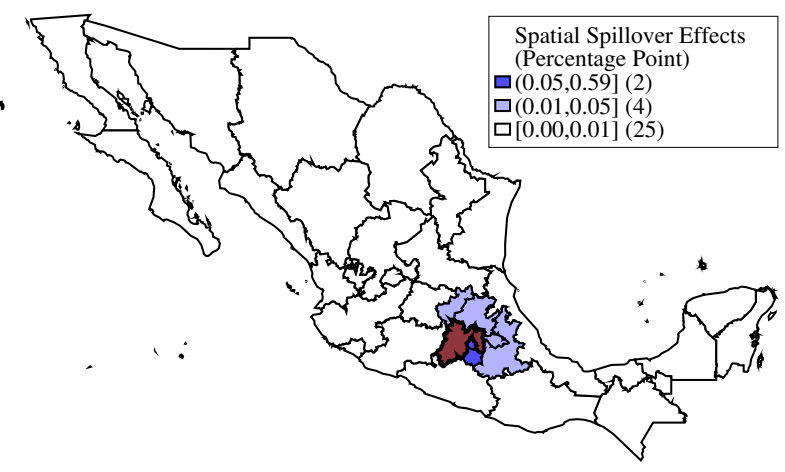

(d) Mexico

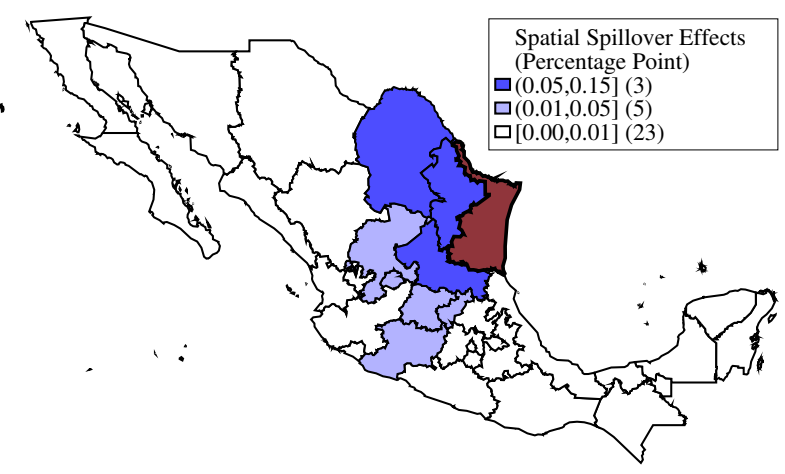

(f) Tamaulipas

Figure 3: Numerical Simulation of Spillover Effects for Selected States

Notes: Author's calculation based on equation (21). 


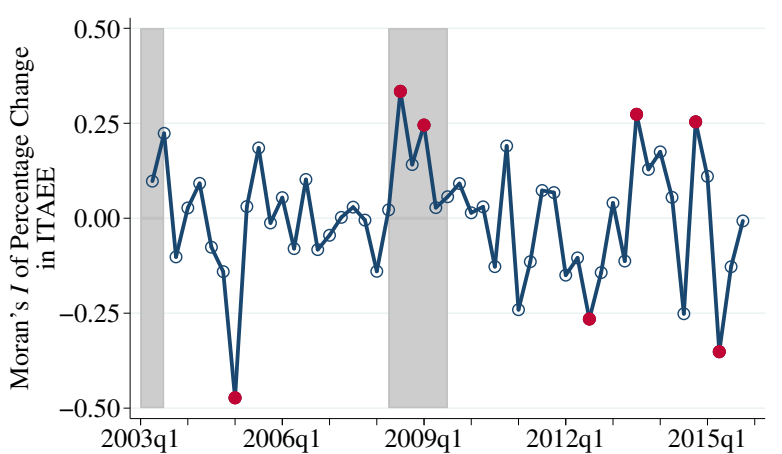

(a) Moran's I in Each Period

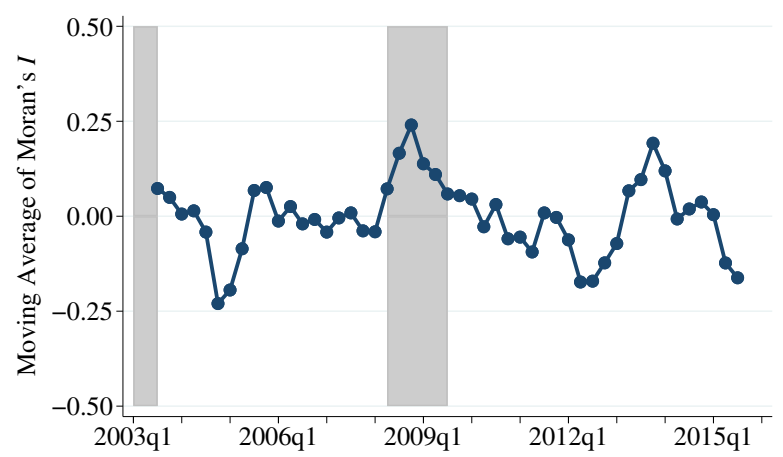

(b) Moving Average of Moran's I

Figure D.1: Time-series of Moran's I

Notes: The variable used for Moran's $I$ is the quarterly growth rate of the Quarterly Indicator of State Economic Activity (Indicador Trimestral de la Actividad Económica Estatal, ITAEE). The red marker indicates statistical significance at the $10 \%$ level. The spatial weight matrix is based on the route distance across states with the distance decay parameter $\eta=4$. Centered moving average of order 3 is calculated in panel (b). 


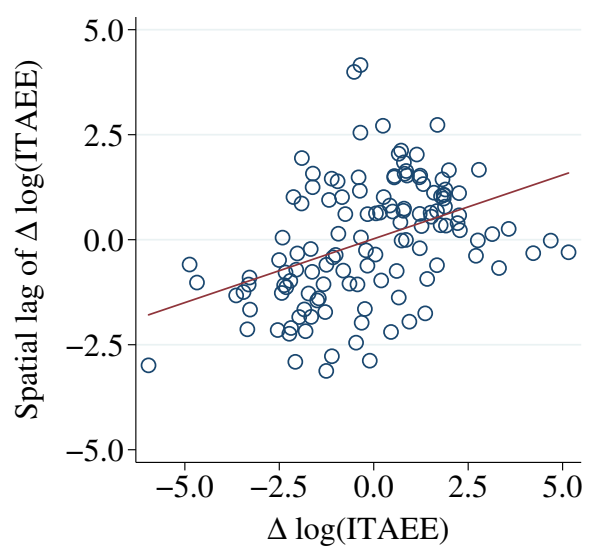

(a) 2008

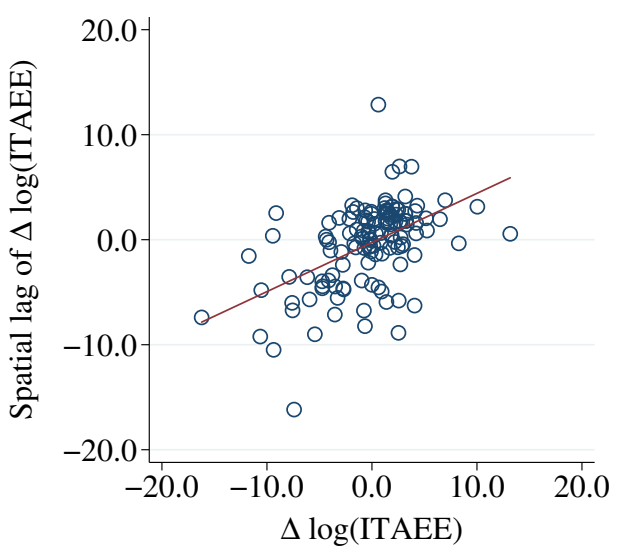

(b) 2009

Figure D.2: Moran Scatter Plot

Notes: The variable is the quarterly growth rate of ITAEE. The spatial weight matrix is based on the route distance across states with the distance decay parameter $\eta=4$. The quarterly data are pooled on a yearly basis. 


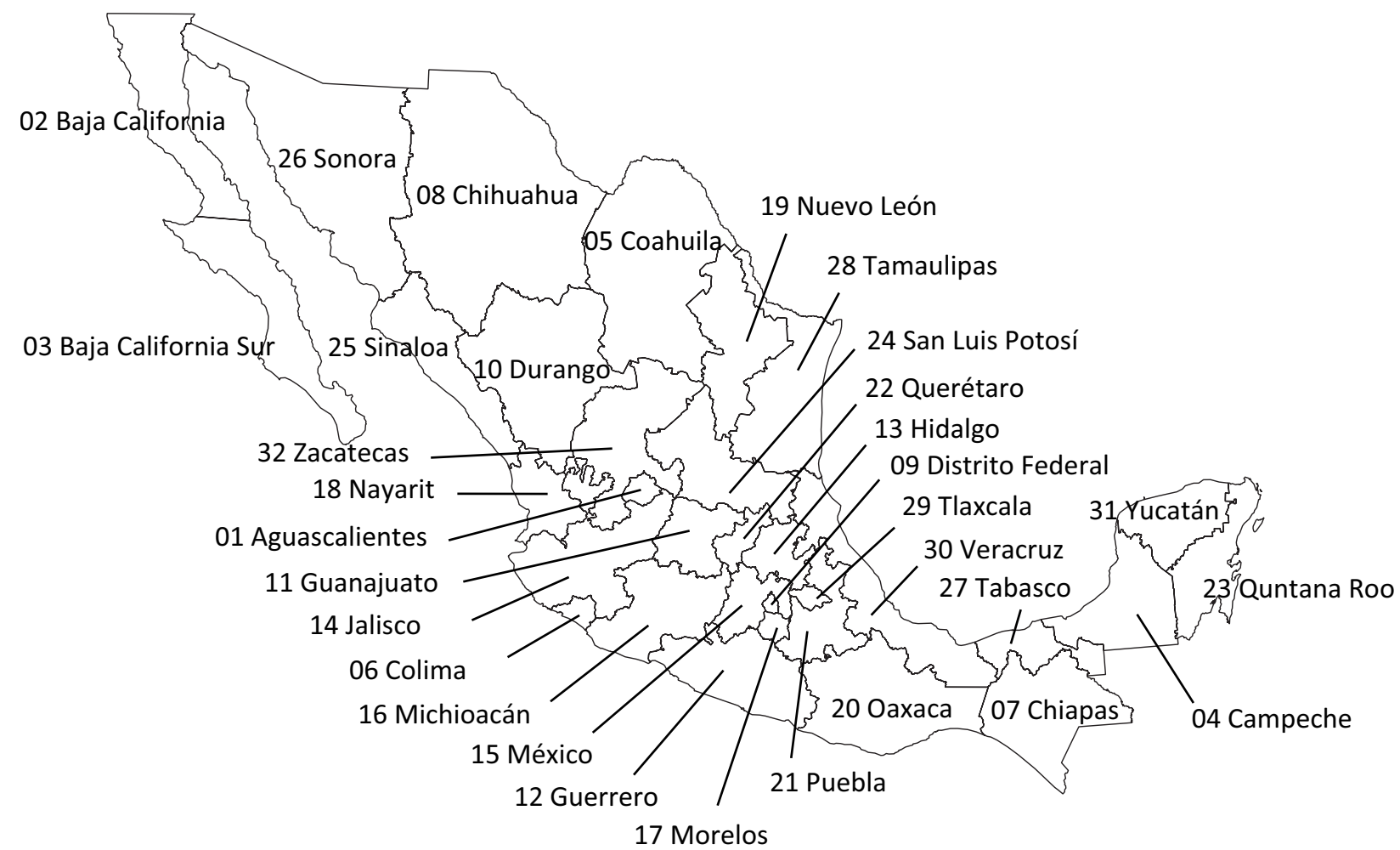

Figure F.1: Map of Mexico 